HETEROCYCLES, Vol. 97, No. 1, 2018, pp. 11 - 42. @ 2018 The Japan Institute of Heterocyclic Chemistry DOI: $10.3987 / C O M-18-S(T)$ Publications

\title{
Professional Publications
}

Kiyoshi Tomioka Professor of Organic Chemistry Faculty of Pharmaceutical Sciences

Doshisha Women's College of Liberal Arts

1. A Biogenetic-Type Asymmetric Synthesis of Natural (+)-Maritidine from L-Tyrosine.

Shun-ichi Yamada, Kiyoshi Tomioka, and Kenji Koga, Tetrahedron Lett., 1976, 57-60.

2. Reductive Decyanization of $\alpha$-Amino Nitriles with Sodium in Liquid Ammonia. Usefulness in Asymmetric Syntheses of Optically Active Alkaloids.

Shun-ichi Yamada, Kiyoshi Tomioka, and Kenji Koga, Tetrahedron Lett., 1976, 61-64.

3. Approaches to the Biogenetic-Type Asymmetric Synthesis of Some Amaryllidaceae Alkaloids.

Kiyoshi Tomioka, Kimihiro Shimizu, Shun-ichi Yamada, and Kenji Koga, Heterocycles, 1977, 6, 1752-1756.

4. Stereochemical Studies. XLIX. A Biogenetic-Type Total Synthesis of Natural (+)-Maritidine from L-Tyrosine Using Highly Specific Asymmetric Cyclization.

Kiyoshi Tomioka, Kenji Koga, and Shun-ichi Yamada,

Chem. Pharm. Bull., 1977, 25, 2681-2688.

5. Stereochemical Studies. L. Reductive Decyanization of $\alpha$-Amino Nitriles with Sodium in Liquid Ammonia. An Alternative Method for the Application to the Asymmetric Synthesis of Optically Active Natural Products.

Kiyoshi Tomioka, Kenji Koga, and Shun-ichi Yamada, Chem. Pharm. Bull., 1977, 25, 2689-2691.

6. A Biogenetic-Type Asymmetric Synthesis of Optically Active Amaryllidaceae Alkaloids: $(+)-$ and (-)-Galanthamine from L-Tyrosine.

Kimihiro Shimizu, Kiyoshi Tomioka, Shun-ichi Yamada, and Kenji Koga, Heterocycles, 1977, 8, 227-282.

7. Stereochemical Studies. LIV. A Biogenetic-Type Asymmetric Synthesis of Optically Active Galanthamine from L-Tyrosine.

Kimihiro Shimizu, Kiyoshi Tomioka, Shun-ichi Yamada, and Kenji Koga, Chem. Pharm. Bull., 1978, 26, 3765-3771.

8. Convenient Preparation of $\alpha, \beta$-Unsaturated Aldehydes.

A. I. Meyers, Kiyoshi Tomioka, and Michael P. Fleming, J. Org. Chem., 1978, 43, 3788-3789.

9. Progress Toward the Total Synthesis of Maytansinoids. An Efficient Route to Two Major Precursors (Western-Southern Zone).

A. I. Meyers, Kiyoshi Tomioka, Dennis M. Roland, and Daniel L. Comins, Tetrahedron Lett., 1978, 1375-1378.

10. Studies Directed Towards the Asymmetric Total Synthesis of Antileukemic Lignan LactonesSynthesis of (-)-Podorhizon. 
Kiyoshi Tomioka, Hidemichi Mizuguchi, and Kenji Koga, Tetrahedron Lett., 1978, 4687-4690.

11. Novel Isomerization of Dibenzocyclooctadiene Lignan Lactone — First Synthesis of $( \pm)$-Stegane Kiyoshi Tomioka, Hidemichi Mizuguchi, and Kenji Koga, Tetrahedron Lett., 1979, 1409-1410.

12. Studies Directed Towards the Asymmetric Total Synthesis of Antileukemic Lignan Lactones. Synthesis of Optically Pure Key Intermediate and Its Utility.

Kiyoshi Tomioka and Kenji Koga, Tetrahedron Lett., 1979, 3315-3318.

13. Asymmetric Total Synthesis of Antileukemic Lignans (+)-trans-Burseran and (-)-Isostegane.

Kiyoshi Tomioka, Tsuneo Ishiguro, and Kenji Koga, J. Chem. Soc., Chem. Commun., 1979, 652-653.

14. Stereoselective Total Synthesis of Optically Active trans-and cis-Burseran.

Kiyoshi Tomioka and Kenji Koga, Heterocycles, 1979, 12, 1523-1526.

15. Asymmetric Synthesis of 2-Substituted Cycloalkanecarboxaldehydes.

Shun-ichi Hashimoto, Hiroshi Kogen, Kiyoshi Tomioka, and Kenji Koga, Tetrahedron Lett., 1979, 3009-3012.

16. Synthesis of Maytansine. Its Recent Aspects. (in Japanese)

Kiyoshi Tomioka, J. Synth. Org. Chem. Jpn., 1979, 37, 322-334.

17. An Efficient Chiral Synthesis of (+)-Sesbanine.

Kiyoshi Tomioka and Kenji Koga, Tetrahedron Lett., 1980, 21, 2321-2324.

18. First Asymmetric Total Synthesis of (+)-Steganacin. Determination of Absolute Stereochemistry.

Kiyoshi Tomioka, Tsuneo Ishiguro, and Kenji Koga,

Tetrahedron Lett., 1980, 21, 2973-2976.

19. Asymmetric Synthesis of 1,2-Disubstituted Cycloalkanecarboxaldehydes. Procedure for the Stereoselective Preparation of cis- and trans-Isomer.

Hiroshi Kogen, Kiyoshi Tomioka, Shun-ichi Hashimoto, and Kenji Koga, Tetrahedron Lett., 1980, 21, 4005-4008.

20. Recent Progress in the Asymmetric Synthesis. I.

Asymmetric Alkylation of Carbonyl Compounds. (in Japanese)

Kiyoshi Tomioka and Kenji Koga, Kagaku no Ryoiki, 1980, 34, 369-378.

21. Recent Progress in the Asymmetric Synthesis. II.

Asymmetric Addition Reaction to Carbonyl Compounds. (in Japanese)

Kiyoshi Tomioka and Kenji Koga,

Kagaku no Ryoiki, 1980, 34, 762-768.

22. Recent Progress in the Asymmetric Synthesis. III. Asymmetric Addition to Carbon-Carbon Double Bonds. (in Japanese) 
Kiyoshi Tomioka and Kenji Koga,

Kagaku no Ryoiki, 1980, 34, 920-927.

23. Highly Stereoselective Construction of Chiral Quaternary Carbon: Asymmetric Synthesis of $\beta, \beta$-Disubstituted- $\gamma$-Butyrolactones.

Kiyoshi Tomioka, Youn-Sang Cho, Fuminori Sato, and Kenji Koga, Chemistry Lett., 1981, 1621-1624.

24. Diastereoselective and Enantioselective Synthesis of 1,2-Disubstituted Cycloalkanecarboxaldehydes.

Hiroshi Kogen, Kiyoshi Tomioka, Shun-ichi Hashimoto, and Kenji Koga, Tetrahedron, 1981, 37, 3951-3956.

25. Asymmetric Total Synthesis of Steganacin. (in Japanese)

Kiyoshi Tomioka and Kenji Koga, Kagaku, 1981, 36, 496-498.

26. Synthetic Approaches Toward Verrucarin A. Chiral Synthesis of (-)-Verrucarinolactone.

Kiyoshi Tomioka, Fuminori Sato, and Kenji Koga, Heterocycles, 1982, 17, 311-316.

27. Stereoselective Reactions. V. Design of the Asymmetric Synthesis of Lignan Lactones. Synthesis of Optically Active Podorhizon and Deoxypodorhizon by 1,3-Asymmetric Induction.

Kiyoshi Tomioka, Hidemichi Mizuguchi, and Kenji Koga, Chem. Pharm. Bull., 1982, 30, 4304-4313.

28. An Asymmetric Synthesis of cis, anti, cis-Tricyclo[5.3.0.0. $\left.{ }^{2,6}\right]$ Decanes Applying $\gamma-$ Hydroxymethyl- $\gamma$-butyrolactone as a Chiral Synthon. First Asymmetric Total Synthesis of (-)- $\beta$ Bourbonene.

Kiyoshi Tomioka, Masahide Tanaka, and Kenji Koga, Tetrahedron Lett., 1982, 23, 3401-3404.

29. Asymmetric Synthesis via Chelate Formation. (in Japanese)

Kiyoshi Tomioka and Kenji Koga, Chemistry Today, 1982, 6, 44-48.

30. Asymmetric Michael Reaction and Its Application. (in Japanese)

Kenji Koga and Kiyoshi Tomioka, Progress in the Asymmetric Synthesis and Resolution, 1982, 97, 33-48.

31. Organic Synthesis by the Use of Metal Cation Capture. (in Japanese)

Kiyoshi Tomioka, Farumashia, 1982, 18, 827-833.

32. Total Synthesis of (-)-Maysine.

A. I. Meyers, Kevin A. Babiak, Arthur L. Campbell, Daniel L. Comins, Michael P. Fleming, Rainer Henning, Manfred Heuschmann, J. P. Hudspeth, J. M. Kane, Paul J. Reider, Dennis M. Roland, Kimihiro Shimizu, Kiyoshi Tomioka, and Robert D. Walkup, J. Am. Chem. Soc., 1983, 105, 5015-5024.

33. Noncatalytic Additions to $\alpha, \beta$-Unsaturated Carbonyl Compounds.

Kiyoshi Tomioka and Kenji Koga, Asymmetric Synthesis, ed. by J. D. Morrison, Academic Press, 1983, Vol. 2, Chapter 7, pp. 201-224. 
34. Asymmetric Total Synthesis of Antileukemic Sesquiterpene (+)-Ivalin.

Kiyoshi Tomioka, Fumio Masumi, Toyoharu Yamashita, and Kenji Koga, Tetrahedron Lett., 1984, 25, 333-336.

35. Conjugate Addition Reaction of Trimethylsilylacetonitrile with $\alpha, \beta$-Unsaturated Carbonyl Compounds. Synthetic Studies Toward Sesbanimide.

Kiyoshi Tomioka and Kenji Koga,

Tetrahedron Lett., 1984, 25, 1599-1600.

36. Asymmetric Alkylation of $\alpha$-Alkyl $\beta$-Keto Esters.

Kiyoshi Tomioka, Kaori Ando, Yutaka Takemasa, and Kenji Koga, J. Am. Chem. Soc., 1984, 106, 2718-2719.

37. Asymmetric Total Synthesis of Natural (-)- and Unnatural (+)-Steganacin. Determination of the Absolute Configuration of Natural Antitumor Steganacin.

Kiyoshi Tomioka, Tsuneo Ishiguro, Yoichi Iitaka, and Kenji Koga, Tetrahedron, 1984, 40, 1303-1312.

38. Factors Controlling the Diastereoface Selectivity in the Complementary Asymmetric Alkylation of $\alpha$-Alkyl $\beta$-Keto Esters.

Kiyoshi Tomioka, Kaori Ando, Yutaka Takemasa, and Kenji Koga, Tetrahedron Lett., 1984, 25, 5677-5680.

39. Asymmetric Total Synthesis of Some Biologically Active Natural Products. (in Japanese)

Kiyoshi Tomioka, Yakugaku Zasshi, 1984, 104, 1009-1023.

40. Structure-Activity Relationships of the Antitumor Compounds. (in Japanese)

Kiyoshi Tomioka, Chemical Industry, 1984, 35, 1049-1054.

41. Stereoselective Reactions. VII. Synthesis of Racemic and Optically pure Isostegane, Isopicrostegane, Picrostegane, and Stegane Employing Novel Isomerization Reaction.

Kiyoshi Tomioka, Hidemichi Mizuguchi, Tsuneo Ishiguro, and Kenji Koga, Chem. Pharm. Bull., 1985, 33, 121-126.

42. Stereoselective Reactions. VIII. Stereochemical Requirement for the Benzylic Oxidation of Lignan Lactone. A Highly Selective Synthesis of the Antitumor Lignan Lactone Steganacin by the Oxidation of Stegane.

Tsuneo Ishiguro, Hidemichi Mizuguchi, Kiyoshi Tomioka, and Kenji Koga, Chem. Pharm. Bull., 1985, 33, 609-617.

43. Enantiospecific Total Synthesis of (-)-Megaphone by a Highly Controlled Consecutive 1,4- and 1,3-Asymmetric Induction.

Kiyoshi Tomioka, Hisashi Kawasaki, and Kenji Koga, Tetrahedron Lett., 1985, 26, 903-906.

44. Enantioselective Conjugate Addition Reaction Mediated by Chiral Ligands.

Kiyoshi Tomioka, Mineichi Sudani, Yuichi Shinmi, and Kenji Koga, Chemistry Lett., 1985, 329-332.

45. Control Elements in the Asymmetric Tandem Alkylation of $\alpha$-Alkylidene- $\gamma$-butyrolactone Derivatives.

Kiyoshi Tomioka, Hisashi Kawasaki, and Kenji Koga, 
Tetrahedron Lett., 1985, 26, 3027-3030.

46. Highly Diastereoselective Tandem Alkylation of Acyclic $\alpha, \beta$-Unsaturated Esters Based on the Novel Use of Dithioacetal Unit.

Hisashi Kawasaki, Kiyoshi Tomioka, and Kenji Koga, Tetrahedron Lett., 1985, 26, 3031-3034.

47. Asymmetric Total Synthesis of Stoechospermol Using Intramolecular [2+2] Photocycloaddition Reaction.

Masahide Tanaka, Kiyoshi Tomioka, and Kenji Koga, Tetrahedron Lett., 1985, 26, 3035-3038.

48. Stereoselective Reactions. X. Stereoselective Total Synthesis of Natural Antitumor Lignan Burseran.

Kiyoshi Tomioka, Tsuneo Ishiguro, and Kenji Koga, Chem. Pharm. Bull., 1985, 33, 4333-4337.

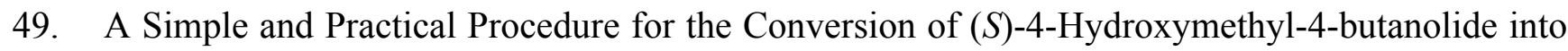
its $(R)$-Enantiomer.

Masahide Tanaka, Kiyoshi Tomioka, and Kenji Koga, Heterocycles, 1985, 23, 2347-2350.

50. The First Total Synthesis of (+)-Spatol with Natural Configuration.

Masahide Tanaka, Kiyoshi Tomioka, and Kenji Koga, Tetrahedron Lett., 1985, 26, 6109-6112.

51. Stereoselective Reactions. XII. Synthesis of Antitumor Active Steganacin Analogs, Picrosteganol and Epipicrosteganol, by Selective Isomerization Reaction.

Kiyoshi Tomioka, Tsuneo Ishiguro, Yoichi Iitaka, and Kenji Koga, Chem. Pharm. Bull., 1985, 34, 1501-1504.

52. Asymmetric Total Synthesis of Antitumor Natural Products. (in Japanese)

Kiyoshi Tomioka, Advances in Pharmaceutical Sciences. The Research Foundation for Pharmaceutical Sciences, 1985, I, 121-137.

53. A New Method for the Diastereoselective Alkylation of Chiral Ester Enolate. (in Japanese) Hisashi Kawasaki, Kiyoshi Tomioka, and Kenji Koga, Kagaku, 1985, 40, 542-543.

54. Asymmetric Conjugate Addition Reaction by the Use of $(S)$ - $\gamma$-Trityloxymethyl- $\gamma$-butyrolactam as a Chiral Auxiliary.

Kiyoshi Tomioka, Toshiro Suenaga, and Kenji Koga, Tetrahedron Lett., 1986, 27, 369-372.

55. Asymmetric Michael Reaction of $\alpha$-Alkyl $\beta$-Keto Esters via Chiral Enamines.

Kiyoshi Tomioka, Kaori Ando, Kosuke Yasuda, and Kenji Koga, Tetrahedron Lett., 1986, 27, 715-718.

56. A Rationale of Diastereofacial Selection in the Alkylation of Endocyclic Enolates with Chirality at the $\beta$-Position.

Kiyoshi Tomioka, Kosuke Yasuda, Hisashi Kawasaki, and Kenji Koga,

Tetrahedron Lett., 1986, 27, 3247-3250. 
57. Enantioface Differentiating Michael Reaction of Ethyl Acetoacetate with Alkylidenemalonates via Chiral Enamine.

Kiyoshi Tomioka, Kosuke Yasuda, and Kenji Koga, Tetrahedron Lett., 1986, 27, 4611-4614.

58. Asymmetric Control in the Construction of Quaternary Carbon Centers and Its Application to the Total Synthesis of Natural Products. (in Japanese)

Kiyoshi Tomioka and Kenji Koga,

$$
\text { J. Synth. Org. Chem. Jpn., 1986, 44, 545-557. }
$$

59. Stereoselective Alkylation Reaction Based on the Allylic Strain Concept. (in Japanese)

Hisashi Kawasaki, Kiyoshi Tomioka, and Kenji Koga, Kagaku, 1986, 41, 361-365.

60. Novel 3,4-Diarylpyrrolidine-Based Chiral Ligands for the Asymmetric Reaction of Arylmagnesium Bromides with Aldehydes.

Kiyoshi Tomioka, Makoto Nakajima, and Kenji Koga, Chemistry Lett., 1987, 65-68.

61. Synthetic Studies Toward Trichothecene Sesquiterpenes. Synthesis of the Optically Pure Key Intermediate for Calonectrin Using Highly Stereoselective Cyclization.

Kiyoshi Tomioka, Masamichi Sugimori, and Kenji Koga, Chem. Pharm. Bull., 1987, 35, 906-908.

62. Novel Use of Aryloxyaluminum Dichloride in the Chiral Diamine Mediated Asymmetric Addition Reaction of Grignard Reagent to Benzaldehyde.

Kiyoshi Tomioka, Makoto Nakajima, and Kenji Koga, Tetrahedron Lett., 1987, 28, 1291-1292.

63. Construction of Contiguous Quaternary and Tertiary Carbons via the Asymmetric Michael Reaction.

Kiyoshi Tomioka, Kosuke Yasuda, and Kenji Koga, J. Chem. Soc., Chem. Commun., 1987, 1345-1346.

64. Stereochemical Aspects of Asymmetric Diels-Alder Reaction Catalyzed by Chiral Alkoxyaluminum Dichloride.

Hiroyuki Takemura, Nobuyasu Komeshima, Ichiro Takahashi, Shun-ichi Hashimoto, Nobuo Ikota, Kiyoshi Tomioka, and Kenji Koga, Tetrahedron Lett., 1987, 28, 5687-5690.

65. Enantioface Differentiation in the cis-Dihydroxylation of C-C Double Bonds by Osmium Tetroxide with Use of a Chiral Diamine with $D_{2}$-Symmetry.

Kiyoshi Tomioka, Makoto Nakajima, and Kenji Koga, J. Am. Chem. Soc., 1987, 109, 6213-6215.

66. Chlorotrimethylsilane Promoted Asymmetric Michael Reaction of Chiral Enamines of $\alpha$-Alkyl $\beta$-Keto Esters.

Kiyoshi Tomioka, Wonjun Seo, Kaori Ando, and Kenji Koga, Tetrahedron Lett., 1987, 28, 6637-6640.

67. Asymmetric Oxidation of Olefins with Osmium Tetroxide. (in Japanese)

Makoto Nakajima, Kiyoshi Tomioka, and Kenji Koga, Kagaku, 1987, 42, 422-423. 
68. A Model for the Diastereofacial Differentiation in the Alkylation of the Endocyclic Enolates.

Kiyoshi Tomioka, Hisashi Kawasaki, Kosuke Yasuda, and Kenji Koga, J. Am. Chem. Soc., 1988, 110, 3597-3601.

69. Mechanistic Aspects of Asymmetric cis-Dihydroxylation of Olefins with Osmium Tetroxide Employing a $C_{2}$ Symmetric Chiral Diamine.

Kiyoshi Tomioka, Makoto Nakajima, Yoichi Iitaka, and Kenji Koga, Tetrahedron Lett., 1988, 29, 573-576.

70. Stereoselective Reactions. XIII. Total Synthesis of (+)-Sesbanine by a Highly Stereoselective Cycloannelation Reaction.

Kiyoshi Tomioka and Kenji Koga, Tetrahedron, 1988, 44, 4351-4355.

71. Stereoselective Reactions. XIV. Efficient Enantioselective Construction of Quaternary Carbon Centers by the Sequential Dialkylation of $(S)-\gamma$-Trityloxymethyl- $\gamma$-Butyrolactone. Synthesis of Optically Active $\beta, \beta$-Disubstituted $\gamma$-Butyrolactones.

Kiyoshi Tomioka, Youn-Sang Cho, Fuminori Sato, and Kenji Koga, J. Org. Chem., 1988, 53, 4094-4098.

72. Total Synthesis of (+)-Sesbanimide A from D-Glucose.

Kiyoshi Tomioka, Atsushi Hagiwara, and Kenji Koga, Tetrahedron Lett., 1988, 29, 3095-3096.

73. New Grignard Reagents. (in Japanese)

Kiyoshi Tomioka, Organic Chemistry and Metal, Farumashia Review No. 24, 1988, pp. 43-49.

74. Asymmetric Michael Reaction by the Use of a Chiral Enamine. (in Japanese)

Kiyoshi Tomioka and Kenji Koga, Kagaku, 1988, 43, 548-549.

75. Stereoselective Reactions. XV. Total Synthesis of (-)-Ivalin by Employing Asymmetric Double Alkylation Reaction of $\alpha, \beta$-Unsaturated Aldimine.

Kiyoshi Tomioka, Fumio Masumi, Toyoharu Yamashita, and Kenji Koga, Tetrahedron, 1989, 45, 643-650.

76. Stereoselective Reactions. XVI. Total Synthesis of $\beta$-Bourbonene by Employing Asymmetric [2+2] Photocycloaddition Reaction of Chiral Butenolide.

Kiyoshi Tomioka, Masahide Tanaka, and Kenji Koga, Chem. Pharm. Bull., 1989, 37, 1201-1207.

77. Synthesis of the Core Enediyne Structure of Esperamicin-Calichemicin Class of Antitumor Antibiotics.

Kiyoshi Tomioka, Hitoshi Fujita, and Kenji Koga, Tetrahedron Lett., 1989, 30, 851-854.

78. Asymmetric $\alpha$-Alkylation of Cyclohexanone Mediated by Chiral Ligand.

Kiyoshi Tomioka, Mitsuru Shindo, and Kenji Koga, Chem. Pharm. Bull., 1989, 37, 1120-1122.

79. Design, Synthesis, and Antitumor Activity of Steganacin Aza-analogue.

Kiyoshi Tomioka, Yoshihiro Kubota, Hisashi Kawasaki, and Kenji Koga, Tetrahedron Lett., 1989, 30, 2949-2952. 
80. Synthesis and Antitumor Activity of Aza-Podophyllotoxin Analogue.

Kiyoshi Tomioka, Yoshihiro Kubota, and Kenji Koga, Tetrahedron Lett., 1989, 30, 2953-2954.

81. Efficient Stereoselective Synthesis of 2-Aza-4'-demethylepipodophyllotoxin.

Kiyoshi Tomioka, Yoshihiro Kubota, and Kenji Koga, J. Chem. Soc., Chem. Commun., 1989, 1622-1624.

82. A Short Asymmetric Synthesis of Anthracycline Antibiotics.

Kiyoshi Tomioka, Makoto Nakajima, and Kenji Koga, J. Chem. Soc., Chem. Commun., 1989, 1921-1922.

83. Novel Strategy of Using a $C_{2}$ Symmetric Chiral Diether in the Enantioselective Conjugate Addition of an Organolithium to an $\alpha, \beta$-Unsaturated Aldimine.

Kiyoshi Tomioka, Mitsuru Shindo, and Kenji Koga, J. Am. Chem. Soc., 1989, 111, 8266-8268.

84. Chemistry of New Antitumor Compounds. (in Japanese)

Kiyoshi Tomioka and Kenji Koga, Kagaku, 1989, 43, 554-555.

85. Asymmetric Oxidation of Olefins with Osmium Tetroxide. (in Japanese)

Makoto Nakajima, Kiyoshi Tomioka, and Kenji Koga, J. Synth. Org. Chem. Jpn., 1989, 47, 878-888.

86. Novel Synthetic Technology. (in Japanese)

Kiyoshi Tomioka and Kenji Koga, Gekkan Yakuzi, 1989, 31, 2321-2326.

87. Nomenclature of Synthetic Drug. (in Japanese)

Kiyoshi Tomioka, Synthetic Drugs, ed. by H. Yajima and M. Hirobe, Hirokawa, 1989, Chapter 1.

88. Asymmetric Diels-Alder Reaction with Use of (S)-5-(Trityloxymethyl)pyrrolidin-2-one as a Chiral Auxiliary.

Kiyoshi Tomioka, Noriko Hamada, Toshiro Suenaga, and Kenji Koga, J. Chem. Soc., Perkin Trans. 1, 1990, 426-428.

89. Conjugate Addition Reaction of Organolithiums to Naphthalenecarboxylates.

Kiyoshi Tomioka, Mitsuru Shindo, and Kenji Koga,

Tetrahedron Lett., 1990, 31, 1739-1740.

90. Dramatic Enantioselectivity Change in Dihydroxylation of Olefins with Chiral Amine-Osmium Tetroxide Complex.

Kiyoshi Tomioka, Makoto Nakajima, and Kenji Koga,

Tetrahedron Lett., 1990, 31, 1741-1742

91. Asymmetric Synthesis Utilizing External Chiral Ligands.

Kiyoshi Tomioka,

Synthesis, 1990, 541-549.

92. Synthesis of Optically Active Compounds. (in Japanese)

Kiyoshi Tomioka,

Experimental Organic Synthesis, Maruzen, 1990. 
93. Reductive Generation of Aldehyde Metal Enolate from Ketene. A One-Flask Process to 1,1,2and 1,2,2-Trisubstituted Dihydronaphthalenes from 1- and 2-Naphthalenecarboxylates.

Kiyoshi Tomioka, Mitsuru Shindo, and Kenji Koga, J. Org. Chem., 1990, 55, 2276-2277.

94. Stereoselective Reactions. XVIII. Synthesis and Cytotoxicity of 10- and 11-Demethylsteganes.

Kiyoshi Tomioka, Hisashi Kawasaki, and Kenji Koga, Chem. Pharm. Bull., 1990, 38, 1899-1901.

95. Stereoselective Reactions. XIX. Asymmetric Dihydroxylation of Olefins by Employing Osmium Tetroxide-Chiral Amine Complexes.

Kiyoshi Tomioka, Yuichi Shinmi, Kunihiko Shiina, Makoto Nakajima, and Kenji Koga, Chem. Pharm. Bull., 1990, 38, 2133-2136.

96. Conjugate Addition to Naphthalenes. (in Japanese)

Mitsuru Shindo, Kiyoshi Tomioka, and Kenji Koga, Kagaku, 1990, 44, 554-555.

97. Asymmetric Organolithium Additions to Imines.

Kiyoshi Tomioka, Isao Inoue, Mitsuru Shindo, and Kenji Koga, Tetrahedron Lett., 1990, 31, 6681-6684.

98. The Most Potent Anticancer Agents. (in Japanese)

Kiyoshi Tomioka, Kagaku, 1990, 44, 768-769.

99. Absolute Structure-Cytotoxic Activity Relationships of Steganacin Congeners and Analogues. Kiyoshi Tomioka, Tsuneo Ishiguro, Hidemichi Mizuguchi, Nobuyoshi Komeshima, Kenji Koga, Shigeru Tsukagoshi, Takashi Tsuruo, Tazuko Tashiro, Seiichi Tanida, and Toyokazu Kishi, J. Med. Chem., 1991, 34, 54-57.

100. An Expeditious Total Synthesis of Dolastatin 10.

Kiyoshi Tomioka, Motomu Kanai, and Kenji Koga, Tetrahedron Lett., 1991, 32, 2395-2398.

101. Catalytic Asymmetric Addition of Organolithiums to Imines.

Kiyoshi Tomioka, Isao Inoue, Mitsuru Shindo, and Kenji Koga, Tetrahedron Lett., 1991, 32, 3095-3098.

102. A Catalytic Method for Asymmetric Nucleophilic Aromatic Substitution Giving Binaphthyls. Mitsuru Shindo, Kenji Koga, and Kiyoshi Tomioka, J. Am. Chem. Soc., 1992, 114, 8732-8733.

103. Enantioselective Conjugate Addition of Organocuprate Using a Chiral Amidophosphine Ligand. Motomu Kanai, Kenji Koga, and Kiyoshi Tomioka Tetrahedron Lett., 1992, 33, 7193-7196.

104. Asymmetric Oxidation. (in Japanese)

Kiyoshi Tomioka, Jikken Kagaku Kouza, 26, pp. 1-22, 1992 (Maruzen).

105. Organic Chemistry, P. S. Bailey, Jr. and C. A. Bailey. (Book, Translation in Japanese) Kenji Koga and Kiyoshi Tomioka, 
Tokyo Kagaku Dojin, 1992.

106. Asymmetric Addition of Aryllithiums to Naphthalene BHA-Esters Catalyzed by a Chiral Ligand. Kiyoshi Tomioka, Mitsuru Shindo, and Kenji Koga, Tetrahedron Lett., 1993, 34, 681-682.

107. Stereoselective Reactions. XXI. Asymmetric Alkylation of $\alpha$-alkyl $\beta$-Keto Esters to $\alpha, \alpha$-Alkyl $\beta$ Keto Esters Having $(R)$ - or $(S)$-Chiral Quaternary Center Depending on the Solvent System.

Kaori Ando, Yutaka Takemasa, Kiyoshi Tomioka, and Kenji Koga, Tetrahedron, 1993, 49, 1579-1588.

108. Design, Synthesis, and Antitumor Activity-Absolute Configuration Relationships of Podophyllotoxin Aza-Analogues. (Stereoselective Reactions. XXII)

Kiyoshi Tomioka, Yoshihiro Kubota, and Kenji Koga Tetrahedron, 1993, 49, 1891-1900.

109. Design, Synthesis, and Structure-Cytotoxicity Relationships of Aza-Steganes (Stereoselective Reactions. XXIII)

Yoshihiro Kubota, Hisashi Kawasaki, Kiyoshi Tomioka, and Kenji Koga, Tetrahedron, 1993, 49, 3081-3090.

110. Solvent Effect and NMR Behaviour in a Chiral Amidophosphine Mediated Reaction of Organocuprate with Chalcone.

Motomu Kanai, Kenji Koga, and Kiyoshi Tomioka, J. Chem. Soc., Chem. Commun., 1993, 1248-1249.

111. Steric Tuning in Chiral Ligand-Catalyzed Enantioselective Alkylation of Imines.

Kiyoshi Tomioka, Isao Inoue, Mitsuru Shindo, and Kenji Koga, Tetrahedron: Asymmetry, 1993, 4, 1603-1606.

112. Reinvestigation of Optical Purities of Optically Active Trimethylsilyl Enol Ethers of 4Substituted Cyclohexanones.

Kazumasa Aoki, Makoto Nakajima, Kiyoshi Tomioka, and Kenji Koga, Chem. Pharm. Bull., 1993, 41, 994-996.

113. Enantioselective Deprotonation of 4-Substituted Cyclohexanones by Chiral Chelated Lithium Amides Having A Fluorine-containing Alkyl Group on Amide Nitrogen.

Kazumasa Aoki, Hiroshi Noguchi, Kiyoshi Tomioka, and Kenji Koga, Tetrahedron Lett., 1993, 34, 5105-5108.

114. Design and Synthesis of Novel Chiral Pyrrolidine-based Diamines with C2-Symmetry.

Makoto Nakajima, Kiyoshi Tomioka, and Kenji Koga, Tetrahedron, 1993, 49 (43), 9735-9750.

115. Enantioselective 1,2-Addition of Grignard Reagents to Aldehydes Using Chiral Diamines. Makoto Nakajima, Kiyoshi Tomioka, and Kenji Koga, Tetrahedron, 1993, 49 (43), 9751-9758.

116. Highly Enantioselective Dihydroxylation of Olefins by Osmium Tetroxide with Chiral Diamines. Makoto Nakajima, Kiyoshi Tomioka, Yoichi Iitaka, and Kenji Koga, Tetrahedron, 1993, 49 (47), 10793-10806.

117. Short-Step Asymmetric Syntheses of Anthracycline Antibiotics via Enantioselective Dihydroxylation by Osmium Tetroxide with a Chiral Diamine. 
Makoto Nakajima, Kiyoshi Tomioka, and Kenji Koga, Tetrahedron, 1993, 49 (47), 10807-10816.

118. Control of Reaction and Stereochemistry. (in Japanese)

Kiyoshi Tomioka, Farumasia, 1993, 29, 168-170.

119. Stereoselective Alkylation Based on the Conformation Control. (in Japanese)

Kiyoshi Tomioka, J. Synth. Org. Chem. Jpn., 1993, 51 (12), 1116-1123.

120. Molecular Basis for Antitumor Activity of Azapodophyllotoxin.

Kiyoshi Tomioka, Mem. Inst. Sci. Ind. Res., Osaka Univ., 1993, 50, 39-44.

121. Complexation of Nucleotides by Flexible Molecular Tweezers Using Pi-Interaction and Electrostatic Interaction.

Shunro Yamaguchi, Yoshio Takai, Masami Sawada, and Kiyoshi Tomioka, Nucleic Acids Symposium Series, 1993, 29, 55-56.

122. A Guide for Experimental Organic Chemistry. (Book in Japanese)

Kiyoshi Tomioka, Masako Nakagawa, Yasushi Yamada, and Hiroto Nagaoka, Hirokawa, 1993.

123. Asymmetric Dihydroxylation Using Osmium Tetroxide. (Book in Japanese)

Kiyoshi Tomioka,

Kikan Kagaku Sosetsu, Asymmetric Synthesis Using Organometallic Reagents, 103-113, Gakkai Syuppan Center, 1993.

124. Asymmetric 1,2-Addition of Organolithiums to Aldimines Catalyzed by Chiral Ligand.

Isao Inoue, Mitsuru Shindo, Kenji Koga, and Kiyoshi Tomioka,

Tetrahedron, 1994, 50 (15), 4429-4438.

125. Stereoselective Reactions. XXII. Asymmetric Michael Reaction of $\alpha$-Alkyl $\beta$-Keto Esters via Chiral Enamines. Dependence of the Diastereoface Selection on the Combination of Solvents and Additives.

Kaori Ando, Kousuke Yasuda, Kiyoshi Tomioka, and Kenji Koga, J. Chem. Soc., Perkin Trans I, 1994, (1), 277-282.

126. Asymmetric Conjugate Addition of Organocopper-Amidophosphine Reagents to Cycloalkenones.

Motomu Kanai and Kiyoshi Tomioka, Tetrahedron Lett., 1994, 35 (6), 895-898.

127. Origin of Regioselectivity in Electrophilic Reaction of Ambident Enaldimines.

Kiyoshi Tomioka, Tetsuji Okamoto, Motomu Kanai, and Hiroshi Yamataka, Tetrahedron Lett., 1994, 35 (12), 1891-1892.

128. Chemical Reactions. (in Japanese)

Kiyoshi Tomioka,

Kinki Kagaku Kougyoukai, 1994, (2), 490, 2-3.

129. Stereo- and Regiochemical Aspects of the Mitsunobu Reaction in Synthesis of Chiral Amino Ether Ligands for Asymmetric Reactions. 
Manabu Okuda and Kiyoshi Tomioka,

Tetrahedron Lett., 1994, 35 (26), 4585-4586.

130. Enantioselective Total Synthesis of $(+)$-Stoechospermol Via Stereoselective Intramolecular (2+2) Photocycloaddition of the Chiral Butenolide.

Masahide Tanaka, Kiyoshi Tomioka, and Kenji Koga, Tetrahedron, 1994, 50 (45), 12829-12842.

131. Total Synthesis of Natural (+)-Spatol. Confirmation of The Absolute Stereostructure.

Masahide Tanaka, Kiyoshi Tomioka, and Kenji Koga, Tetrahedron, 1994, 50 (45), 12843-12852.

132. Chlorotrimethylsilane Promoted Asymmetric Michael Reaction of Chiral Lithioenamines Derived from $\alpha$-Alkyl $\beta$-Keto Esters.

Kaori Ando, Wonjun Seo, Kiyoshi Tomioka, and Kenji Koga, Tetrahedron, 1994, 50 (46), 13081-13088.

133. Heterogeneous Fluorination of Allylic Halides by the Combination of Lead Fluoride and Sodium Salts.

Junko Ichihara, Yoshio Takai, Terukiyo Hanafusa, and Kiyoshi Tomioka, J. Fluorine Chem., 1995, 71, 131-133.

134. Highly Enantioselective Aldol Reaction Mediated by Mixed Aggregates Derived from a Lithium Ester Enolate and Tetradentate Chiral Lithium Amides.

Maki Uragami, Kiyoshi Tomioka, and Kenji Koga, Tetrahedron: Asymmetry, 1995, 6 (3), 701-704.

135. Diastereoselective Thiophenol Addition to $(S)-N$ - $\alpha, \beta$-Unsaturated Carbonyl- $\gamma$-trityloxymethyl- $\gamma$ butyrolactams.

Kiyoshi Tomioka, Aki Muraoka, and Motomu Kanai, J. Org. Chem., 1995. 60 (19), 6188-6190.

136. Organic chemistry is quite wide. (in Japanese)

Kiyoshi Tomioka, Kagaku, 1995, 50 (4), 200-202,

137. Asymmetric Conjugate Addition of Organomagnesium Cuprates Controlled by Stoichiometric Amount of Chiral Phosphine.

Motomu Kanai and Kiyoshi Tomioka, Tetrahedron Lett., 1995, 36 (24), 4273-4274.

138. Catalytic Asymmetric Conjugate Addition of Grignard Reagents Controlled by Copper(I)-Chiral Bidentate Phosphine Complex.

Motomu Kanai and Kiyoshi Tomioka, Tetrahedron Lett., 1995, 36 (24), 4275-4278.

139. Fine Chemicals. (in Japanese)

Kiyoshi Tomioka Co-ed., Kagaku-Binran, Applied Chemistry 5th, Chapter 10, 137-327, Maruzen, 1995.

140. Asymmetric Conjugate Addition Reaction of Organocopper Based on Metal Differentiating Coordination. (in Japanese)

Motomu Kanai and Kiyoshi Tomioka, Organometallic News, 1995, 2, 44-49. 
141. Chiral Environment and Synthesis of Chiral Molecule. (in Japanese)

Kiyoshi Tomioka,

Seisan to Gijutu, 1995, 47 (3), 57-59.

142. Gakusiin Prize Award Research by Professor Kenji Koga. (in Japanese)

Kiyoshi Tomioka,

J. Synth. Org. Chem. Jpn., 1995, 53 (7), 565.

143. Selective Synthesis of Enantiomer-Concept in Asymmetric Synthesis. (in Japanese)

Kiyoshi Tomioka,

Chemistry and Education, 1995, 43 (11), 700-702.

144. Enantioselective Reaction of An Imine with Methyllithium Catalyzed by A Chiral Ligand.

Isao Inoue, Mitsuru Shindo, Kenji Koga, Motomu Kanai, and Kiyoshi Tomioka, Tetrahedron: Asymmetry, 1995, 6 (10), 2527-2533.

145. Conformational Preference and Diastereoselectivity of $(S)-N-(\alpha, \beta$-Unsaturated Carbonyl)- $\gamma$ trityloxymethyl- $\gamma$-butyrolactam.

Motomu Kanai, Aki Muraoka, Takanori Tanaka, Masami Sawada, Nobuo Ikota, and Kiyoshi Tomioka,

Tetrahedron Lett., 1995, 36 (51), 9349-9352

146. Theoretical Calculation-Based Reproduction of Thermodynamic, Kinetic Behaviors and Cytotoxicity of Azasteganes. (Special Issue for the Memory of Prof. Yoshio Ban)

Kiyoshi Tomioka, Motomu Kanai, and Nobuo Ikota

Heterocycles, 1996, 42 (1), 43-45.

147. Asymmetric Conjugate Addition Reaction of Organometallic Reagents. (Review in Japanese)

Motomu Kanai and Kiyoshi Tomioka, Kagaku, 1996, 51 (1), 64-65.

148. Catalytic Asymmetric Conjugate Addition Reaction of Organocopper. (Review in Japanese)

Motomu Kanai and Kiyoshi Tomioka, Farumasia, 1996, 32 (2), 171-174.

149. Catalytic Asymmetric Conjugate Addition Reaction of Organocopper Reagents. (Review in Japanese)

Motomu Kanai, Yuichi Nakagawa, and Kiyoshi Tomioka, J. Synth. Org. Chem. Jpn., 1996, 54 (6), 474-480.

150. Standardization of Word in Pharmaceutical Sciences. (in Japanese)

Kiyoshi Tomioka,

Life and Pharmaceuticals, 1996, 12 (4), 1.

151. Chiral Ligand Controlled Enantioselective Opening of Oxirane and Oxetane.

Masashi Mizuno, Motomu Kanai, Akira Iida, and Kiyoshi Tomioka, Tetrahedron: Asymmetry, 1996, 7 (9), 2483-2484.

152. Structural Requirements of External, Chiral Amidophosphine Ligand for Asymmetric Reaction of Organocopper Reagent.

Yuichi Nakagawa, Motomu Kanai, Yasuo Nagaoka, and Kiyoshi Tomioka, Tetrahedron Lett., 1996, 37 (43), 7805-7808.

153. Trichothecinols A, B and C, Potent Anti-Tumor Promoting Sesquiterpenoids from the Fungus 
Trichothecium roseum.

Akira Iida, Kazuhide Konishi, Hiroki Kubo, Kiyoshi Tomioka, Harukuni Tokuda, and Hoyoku Nishino,

Tetrahedron Lett., 1996, 37 (51), 9219-9220.

154. A Ternary Complex-Reagent for An Asymmetric Reaction of Lithium Ester Enolates with Imines

Hiroki Fujieda, Motomu Kanai, Takeshi Kambara, Akira Iida, and Kiyoshi Tomioka J. Am. Chem. Soc., 1997, 119 (8), 2060-2061.

155. Enantioselective Addition of Methyllithium to 2-Furaldehyde Imine with the Aid of Chiral Ligand. (Special Issue for the Memory of the late Professor Shun-ichi Yamada)

Daisuke Taniyama, Motomu Kanai, Akira Iida, and Kiyoshi Tomioka, Heterocycles, 1997, 46, 165-168.

156. Asymmetric Addition Reaction of Phenyllithium to 1,2-Ethylenediimine with The Aid of A Chiral Ligand.

Daisuke Taniyama, Motomu Kanai, Akira Iida, and Kiyoshi Tomioka, Chem. Pharm. Bull., 1997, 45 (10), 1705-1707.

157. An External Chiral Ligand Controlled Enantioselective Opening of Oxirane and Oxetane By Organolithiums.

Masashi Mizuno, Motomu Kanai, Akira Iida, and Kiyoshi Tomioka, Tetrahedron, 1997, 53 (31), 10699-10708.

158. Stereoselective Reactions. 28. Effects of the Alkyl Group at the Amide Nitrogen of Chiral Bidentate Lithium Amides on Enantioselective Deprotonation Reaction.

Kazumasa Aoki, Kiyoshi Tomioka, Hiroshi Noguchi, and Kenji Koga, Tetrahedron, 1997, 53 (40), 13641-13656.

159. Targeting DNA Topoisomerase II with Podophyllotoxin Aza-Analogue.

Akira Iida, Masahiro Kano, Yoshihiro Kubota, Kenji Koga, and Kiyoshi Tomioka, Bioorg. Med. Chem. Lett., 1997, 7 (20), 2565-2566.

160. Enantioselective Conjugate Additions of Organolithiums to BHA Enoates Mediated By A Chiral Ligand.

Yasutomi Asano, Akira Iida, and Kiyoshi Tomioka, Tetrahedron Lett., 1997, 38 (52), 8973-8976.

161. Steric Tuning of Reactivity and Enantioselectivity in Addition of Thiophenol to $\alpha, \beta$-Unsaturated Esters Mediated by a Chiral Ligand.

Katsumi Nishimura, Masashi Ono, Yasuo Nagaoka, and Kiyoshi Tomioka, J. Am. Chem. Soc., 1997, 119 (52), 12974-12975.

162. Enantioselective Addition of Thiazolyllithium to Aldimines with the Aid of Chiral Ligand. Asymmetric Synthesis of (S)-Doe, A Component of Marine Natural Product, Dolastatin 10. (Professor Koji Nakanishi's Special issue)

Kiyoshi Tomioka, Maki Satoh, Daisuke Taniyama, Motomu Kanai, and Akira Iida. Heterocycles, 1998, 47, 77-81.

163. The Asymmetric Horner-Wadsworth-Emmons Reaction Mediated by An External Chiral Ligand.

Masashi Mizuno, Kunihiko Fujii, and Kiyoshi Tomioka, Angew. Chem., Int. Ed. Engl., 1998, 37 (4), 515-517. 
164. The Chiral Ligand Catalyzed Enantioselective Conjugate Addition of Organolithium to BHA Enoates.

Yasutomi Asano, Akira Iida, and Kiyoshi Tomioka, Chem. Pharm. Bull., 1998, 46 (1), 184-186.

165. Structural Requirements of a Chiral Ligand for the Catalytic Asymmetric Addition of Thiophenol to $\alpha, \beta$-Unsaturated Esters.

Kiyoshi Tomioka, Manabu Okuda, Katsumi Nishimura, Shino Manabe, Motomu Kanai, Yasuo Nagaoka, and Kenji Koga,

Tetrahedron Lett., 1998, 39 (15), 2141-2144.

166. An External Chiral Amidophosphine Ligand for Asymmetric Conjugate Addition of Organocopper.

Yuichi Nakagawa, Motomu Kanai, Yasuo Nagaoka, and Kiyoshi Tomioka, Tetrahedron, 1998, 54 (35), 10295-10307.

167. Baylis-Hillman Type Carbon-Carbon Bond Formation of Alkenylphosphonates by the Action of Lithium Diisopropylamide.

Yasuo Nagaoka and Kiyoshi Tomioka, J. Org. Chem., 1998, 63 (19), 6428-6429.

168. Enantioselective Conjugate Addition of Diethylzinc to Cyclohexenone Catalyzed by A Chiral Aminophosphine-Copper(II) Triflate.

Toshiyuki Mori, Kyoko Kosaka, Yuichi Nakagawa, Yasuo Nagaoka, and Kiyoshi Tomioka, Tetrahedron: Asymmetry, 1998, 9 (18), 3175-3178.

169. Design, Synthesis, and Application of $\mathrm{A} \mathrm{C}_{2}$ Symmetric Chiral Ligand for Enantioselective Conjugate Addition of Organolithium to $\alpha, \beta$-Unsaturated Aldimine.

Mitsuru Shindo, Kenji Koga, and Kiyoshi Tomioka, J. Org. Chem., 1998, 63 (25), 9351-9357.

170. A Factor Affecting Enantioselective Reaction of a Lithium Ester Enolate Ternary Complex With Imine.

Takeshi Kambara, Mostafa Ahmed Hussein, Hiroki Fujieda, Akira Iida, and Kiyoshi Tomioka, Tetrahedron Lett., 1998, 39 (49), 9055-9058.

171. Asymmetric Synthesis. Graphical Abstracts and Experimental Methods.

Tamio Hayashi, Kiyoshi Tomioka, and Osamu Yonemitsu (Co ed.),

Kodansha (and Gordon and Breach Science Publishers), 1998.

172. Awardee of Pharmaceutical Society Japan, Dr. Shiro Terashima. (in Japanese)

Kiyoshi Tomioka, Farumashia, 1998, 34 (4), 373.

173. Retrosynthesis. (Book in Japanese)

Kiyoshi Tomioka co-author.

Organic Chemistry for Graduate Course, Tokyo Kagaku Dojin, 1998.

174. Organic Synthesis by Christine Willis and Martin Wills. (Book, Translation in Japanese) Kiyoshi Tomioka, Kagakudojin, 1998.

175. The Conjugate Addition-Aldol Tandem Reaction of $\alpha, \beta$-Unsaturated Esters Catalyzed By Lithium Benzenethiolate. 
Masashi Ono, Katsumi Nishimura, Yasuo Nagaoka, and Kiyoshi Tomioka, Tetrahedron Lett., 1999, 40 (8), 1509-1512.

176. A Facile Asymmetric Synthesis of 1-Substituted Tetrahydroisoquinoline Based on A Chiral Ligand-Mediated Addition of Organolithium to Imine.

Daisuke Taniyama, Masayoshi Hasegawa, and Kiyoshi Tomioka, Tetrahedron: Asymmetry, 1999, 10 (2), 221-224.

177. An Asymmetric Conjugate Addition Reaction of Lithium Organocopper Reagent Controlled by A Chiral Amidophosphine.

Motomu Kanai, Yuichi Nakagawa, and Kiyoshi Tomioka, Tetrahedron, 1999, 55 (13), 3831-3842.

178. A Catalytic Enantioselective Conjugate Addition of Grignard Reagents to Cyclic $\alpha, \beta-$ Unsaturated Carbonyl Compounds Catalyzed by A Chiral Amidophosphine-Copper Iodide.

Motomu Kanai, Yuichi Nakagawa, and Kiyoshi Tomioka, Tetrahedron, 1999, 55 (13), 3843-3854.

179. A One-Flask Synthesis of Dihydronaphthalenemethanols by Directed Addition of Organolithium Reagents to BHA Naphthalenecarboxylates.

Mitsuru Shindo, Kenji Koga, Yasutomi Asano, and Kiyoshi Tomioka, Tetrahedron, 1999, 55 (16), 4955-4968.

180. Catalytic Asymmetric Reaction of Lithium Ester Enolate with Imine.

Kiyoshi Tomioka, Hiroki Fujieda, Satoko Hayashi, Mostafa Ahmed Hussein, Takeshi Kambara, Yumiko Nomura, Motomu Kanai, and Kenji Koga, Chem. Commun., 1999, (8), 715-716.

181. Unexpected Catalytic Behavior of Chiral Bisoxazoline in Asymmetric Reaction of Lithium Ester Enolate with Imine.

Takeshi Kambara and Kiyoshi Tomioka, Chem. Pharm. Bull, 1999, 47 (5), 720-721.

182. Asymmetric Protonation of Lithium Enolate Using 5-Substituted Pyrrolidin-2-One as A Chiral Proton Source.

Hidetaka Fujihara and Kiyoshi Tomioka, J. Chem. Soc., Perkin Trans. 1, 1999, (16), 2377-2382.

183. Synthesis of 1,2-Disubstituted Naphthalenes and Tetrahydronaphthalenes from Dihydronaphthalenes obtained by Conjugate Addition of Organolithium Reagents to BHA Naphthalenecarboxylates.

Mitsuru Shindo, Kenji Koga, and Kiyoshi Tomioka, Chem. Pharm. Bull., 1999, 47 (9), 1318-1321.

184. Efficient Cyclization of $\omega$-Oxo- $\alpha, \beta$-Unsaturated Esters Using Lithium Thiolate-Initiated Michael-Aldol Tandem Reaction.

Masashi Ono, Katsumi Nishimura, Yasuo Nagaoka, and Kiyoshi Tomioka, Tetrahedron Lett., 1999, 40 (38), 6979-6982.

185. Studies Aimed at Enhancement of Reactivity and Enantioselectivity of a Lithium Ester Enolate Using a Tridentate Chiral Lithium Amide.

Mostafa Ahmed Hussein, Akira Iida and Kiyoshi Tomioka, Tetrahedron, 1999, 55 (37), 11219-11228. 
186. Chemoselective Alkoxycarbonylation Reagent Having Trifluoromethanesulfonyl-4trifluoromethylanilide as a Leaving Group.

Tomohisa Yasuhara, Yasuo Nagaoka, and Kiyoshi Tomioka, J. Chem. Soc., Perkin Trans. 1, 1999, (16), 2233-2234.

187. Cyclization of $\alpha, \beta, \psi, \omega$-Unsaturated Bisphosphonates Using Organolithium-Initiated Conjugate Addition-Michael Tandem Reaction.

Yasuo Nagaoka and Kiyoshi Tomioka, Org. Lett., 1999, 1 (9), 1467-1469.

188. Formal Asymmetric Synthesis of Cholesterol Absorption Inhibitor Bearing 2-Azaspiro[3.5]nonan-1-one Moiety.

Takeshi Kambara and Kiyoshi Tomioka, J. Org. Chem., 1999, 64 (25), 9282-9285.

189. Conjugate Addition Reactions.

Kiyoshi Tomioka and Yasuo Nagaoka,

Comprehensive Asymmetric Catalyst Vol. III, chapter 31, ed. by E. N. Jacobsen, A. Pfaltz, H. Yamamoto, Springer, 1999, pp. 1105-1120.

190. The Most Powerful Anticancer Agents. (in Japanese)

Kiyoshi Tomioka, World Records in Chemistry, Kagaku Dojin, 1999.

191. Invitation to our Laboratory. (in Japanese)

Kiyoshi Tomioka, Kagaku, 1999, 54 (10), 45-48.

192. Enantioselective Conjugate Addition of Organometallic Reagents to Cycloalkenones by The Aid of Chiral Lactam-Phosphine Ligand.

Kiyoshi Tomioka and Yuichi Nakagawa, Heterocycles, 2000, 52 (1), 95-97.

193. Approach to Enantioselective Conjugate Addition of Organocopper Reagents to Cycloalkenones by The Aid of Chiral Lactam Bearing Phosphine Group.

Yuichi Nakagawa, Koichiro Matsumoto, and Kiyoshi Tomioka, Tetrahedron, 2000, 56 (18), 2857-2863.

194. Podophyllotoxin Aza-Analogue, A Novel DNA Topoisomerase II Inhibitor.

Akira Iida, Masahiro Kano, Yoshihiro Kubota, Kenji Koga, and Kiyoshi Tomioka, Chem. Pharm. Bull., 2000, 48 (4), 486-489.

195. Facile Asymmetric Synthesis of $\alpha$-Amino Acids Employing Chiral Ligand-Mediated Asymmetric Addition Reactions of Phenyllithium with Imines.

Masayoshi Hasegawa, Daisuke Taniyama, and Kiyoshi Tomioka, Tetrahedron, 2000, 56 (52), 10153-10158.

196. A Facile and Efficient Asymmetric Synthesis of (+)-Salsolidine.

Daisuke Taniyama, Masayoshi Hasegawa, and Kiyoshi Tomioka, Tetrahedron Lett., 2000, 41 (29), 5533-5536.

197. Controlling Factors in Chiral Bisoxazoline-Catalyzed Asymmetric Lithium Ester Enolate-Imine Condensation Producing a $\beta$-Lactam. (Prof. K. Tsuda's memorial)

Takeshi Kambara and Kiyoshi Tomioka, 
Chem. Pharm. Bull., 2000, 48 (10), 1577-1580.

198. An Activated Phosphate for an Efficient Amide and Peptide Coupling Reagent.

Tomohisa Yasuhara, Yasuo Nagaoka, and Kiyoshi Tomioka, J. Chem. Soc., Perkin Trans. 1, 2000, (17), 2091-2092.

199. Copper-Amidophosphine Catalyst in Asymmetric Addition of Organozinc to Imines.

Hidetaka Fujihara, Kazushige Nagai, and Kiyoshi Tomioka, J. Am. Chem. Soc., 2000, 122 (48), 12055-12056.

200. Asymmetric Michael-Type Addition Reaction.

Kiyoshi Tomioka, Modern Carbonyl Chemistry, Chapter 12, ed. by J. Otera, Wiley-VCH, Germany, 2000.

201. Asymmetric Reaction of Organolithium Reagents under Control of Chiral Ligand. (in Japanese) Kiyoshi Tomioka and Masayoshi Hasegawa, J. Synth. Org. Chem. Jpn., 2000, 58 (9), 848-855.

202. New Chiral Amine Ligands for Enantioselective Synthesis of Certain $(S)$ - and $(R)$-Monobactams. M. A. Hussein, A. A. El-Shorbagi, N. M. Omar, Z. S. Farghaly, and Kiyoshi Tomioka, Bull. Pharm. Sci., Assiut Univ., 2000, 23 (2), 125-136.

203. Catalytic Enantioselective Protonation of Lithium Ester Enolates Generated by Conjugate Addition of Arylthiolate to Enoates.

Katsumi Nishimura, Masashi Ono, Yasuo Nagaoka, and Kiyoshi Tomioka, Angew. Chem. Int. Ed., 2001, 40 (2), 440-442.

204. Chiral Ketone-Catalyzed Asymmetric Epoxidation of Stilbene with Oxone. (Prof. Sho Ito's Special issue)

Koichiro Matsumoto and Kiyoshi Tomioka, Heterocycles, 2001, 54, 615-617.

205. Chiral Amidomonophosphine-Rhodium(I) Catalyst for Asymmetric 1,4-Addition of Arylboronic Acids to Cycloalkenones.

Masami Kuriyama and Kiyoshi Tomioka, Tetrahedron Lett., 2001, 42 (5), 921-923.

206. Electronic and Steric Control in Regioselective Addition Reactions of Organolithium Reagents with Enaldimines.

Kiyoshi Tomioka, Yoshito Shioya, Yasuo Nagaoka, and Ken-ichi Yamada, J. Org. Chem., 2001, 66 (21), 7051-7054.

207. Molecular Assembly and Gelating Behaviour of Didodecanoylamides of $\alpha, \omega$-Alkylidenediamines.

Kiyoshi Tomioka, Takaaki Sumiyoshi, Shinobu Narui, Yasuo Nagaoka, Akira Iida, Yoshihisa Miwa, Tooru Taga, Minoru Nakano, and Tetsuro Handa, J. Am. Chem. Soc., 2001, 123 (47), 11817-11818.

208. Total Synthesis of (-)-Neplanocin A Using Lithium Thiolate-Initiated Michael-Aldol Tandem Cyclization Reaction.

Masashi Ono, Katsumi Nishimura, Hiroshi Tsubouchi, Yasuo Nagaoka, and Kiyoshi Tomioka, J. Org. Chem., 2001, 66 (24), 8199-8203. 
209. First Asymmetric Synthesis of a Dopamine D1 Agonist, Dihydrexidine, Employing Asymmetric Conjugate Addition Technology.

Yasutomi Asano, Mitsuaki Yamashita, Kazushige Nagai, Masami Kuriyama, Ken-ichi Yamada, and Kiyoshi Tomioka, Tetrahedron Lett., 2001, 42 (48), 8493-8495.

210. An Approach to a Chiral Cycloalkanone-Mediated Asymmetric Epoxidation of Stilbene with Oxone ${ }^{\circledR}$.

Koichiro Matsumoto and Kiyoshi Tomioka, Chem. Pharm. Bull., 2001, 49 (12), 1653-1657.

211. Awardee of Pharmaceutical Society Japan, Dr. Kazunori Odashima. (in Japanese)

Kiyoshi Tomioka, Farumashia, 2001, 37 (5), 433.

212. Synthesis of Allenes by Horner-Wadsworth-Emmons Reaction.

Hideki Inoue, Hiroshi Tsubouchi, Yasuo Nagaoka, and Kiyoshi Tomioka, Tetrahedron, 2002, 58 (1), 83-90.

213. Chiral Ketone-Catalyzed Asymmetric Epoxidation of Olefins with Oxone.

Koichiro Matsumoto and Kiyoshi Tomioka, Tetrahedron Lett., 2002, 43 (4), 631-633.

214. Synthesis and Application of Chiral Bisphosphines through Lithiation-Conjugate Addition Tandem Cyclization of Chiral $\alpha, \beta, \chi, \psi$-Unsaturated Bisphosphine Oxide.

Yasuo Nagaoka, Hideki Inoue, Nawal El-Kossi, and Kiyoshi Tomioka, Chem. Commun., 2002, (2), 122-123.

215. Efficient Chiral Amidophosphine Ligand for Copper-Catalyzed Asymmetric Addition of Diethylzinc to $\mathrm{N}$-Sulfonylimines. (Special issue for Prof. Mukaiyama)

Kazushige Nagai, Hidetaka Fujihara, Masami Kuriyama, Ken-ichi Yamada, and Kiyoshi Tomioka,

Chemistry Lett., 2002, (1), 8-9.

216. Chiral Amino Ether-Controlled Catalytic Enantioselective Arylthiol Conjugate Addition to $\alpha, \beta-$ Unsaturated Esters and Ketones: Scope, Structural Requirements, and Mechanistic Implications.

Katsumi Nishimura and Kiyoshi Tomioka, J. Org. Chem., 2002, 67 (2), 431-434.

217. Synthesis of Allenes by Horner-Wadsworth-Emmons Reaction.

Yasuo Nagaoka, Hideki Inoue, and Kiyoshi Tomioka, Phosphorus, Sulfur and Silicon and the Related Elements, 2002, 177 (6-7), 1843-1846.

218. Chiral phosphine ligand via cyclization of bis-alkenylphosphonate.

Yasuo Nagaoka, Hideki Inoue, and Kiyoshi Tomioka, Phosphorus, Sulfur and Silicon and the Related Elements, 2002, 177 (8-9), 1959-1960.

219. Chiral Ligand-Controlled Catalytic Asymmetric Epoxidation Of Enone With Hydroperoxide. (A. I. Meyer's Special issue)

Yoshihito Tanaka, Katsumi Nishimura, and Kiyoshi Tomioka, Heterocycles, 2002, 58, 71-73.

220. Conjugate Reduction-Initiated Tandem Cyclization of A Chiral $\alpha, \beta, \chi, \psi-$ Unsaturated Bisphosphine 
Oxide.

Yasuo Nagaoka, Nawal El-Koussi, Shinichi Uesato, and Kiyoshi Tomioka, Tetrahedron Lett., 2002, 43 (24), 4355-4359.

221. Hemilabile Amidomonophosphine Ligand-Rhodium(I) Complex-Catalyzed Asymmetric 1,4Addition of Arylboronic Acids to Cycloalkenones.

Masami Kuriyama, Kazushige Nagai, Ken-ichi Yamada, Yoshinao Miwa, Tooru Taga, and Kiyoshi Tomioka,

J. Am. Chem. Soc., 2002, 124 (30), 8932-8939.

222. A Chiral Ligand-Mediated Asymmetric Addition of A Lithium BHA Ester Enolate To An Aldehyde.

Yumiko Nomura, Mayu Iguchi, Hirohisa Doi, and Kiyoshi Tomioka, Chem. Pharm. Bull., 2002, 50 (8), 1131-1134.

223. A New Methodology for Synthesis of A Chiral Phosphinocarboxylic Acid Through Michael Cyclization-Aldol Tandem Reaction of Chiral $\alpha, \beta, \chi, \psi$-Unsaturated Bisphosphine Oxide and Application in Palladium-Catalyzed Asymmetric Allylic Alkylation.

Hideki Inoue, Yasuo Nagaoka, and Kiyoshi Tomioka, J. Org. Chem., 2002, 67 (16), 5864-5867.

224. Radical Addition of Ethers to Imines Initiated by Dimethylzinc.

Ken-ichi Yamada, Hidetaka Fujihara, Yasutomo Yamamoto, Yoshihisa Miwa, Tooru Taga, and Kiyoshi Tomioka,

Org. Lett., 2002, 4 (20), 3509-3511.

225. External Chiral Ligand-Mediated Enantioselective Peterson Reaction of $\alpha$-Trimethylsilanylacetate with Substituted Cyclohexanones.

Mayu Iguchi and Kiyoshi Tomioka

Org. Lett., 2002, 4 (24), 4329-4331.

226. Chiral Ligand-Controlled Asymmetric Reactions of Organolithium Reagents. Asymmetric Conjugate Addition of Arylthiols to Enoates and its Application to Organic Synthesis of Biologically Potent Compounds.

Kiyoshi Tomioka,

My Favorite Organic Synthesis, 2002, 226-227, The Society of Synthetic Organic Chemistry.

227. Process Chemistry as 21 Century Science. (In Japanese)

Takayuki Shioiri, Shigeru Soda, Kiyoshi Tomioka, Koji Tomimoto, and Kazuma Aoki, Farumasia, 2002, 38 (3), 197-202.

228. Asymmetric Michael-Aldol Tandem Cyclization of $\omega$-Oxo- $\alpha, \beta$-unsaturated Esters with 10Mercaptoisoborneol Methyl Ether.

Katsumi Nishimura, Hiroshi Tsubouchi, Masashi Ono, Tomoharu Hayama, Yasuo Nagaoka, and Kiyoshi Tomioka,

Tetrahedron Lett., 2003, 44 (11), 2323-2326.

229. Chiral Ligand-Controlled Asymmetric Conjugate Addition of Lithium Amides to Enoates.

Hirohisa Doi, Takeo Sakai, Mayu Iguchi, Ken-ichi Yamada, and Kiyoshi Tomioka, J. Am. Chem. Soc., 2003, 125 (10), 2886-2887.

230. Stereoselective Synthesis of The Optically Pure AB-Ring Moiety of Trichothecene Sesquiterpene (+)-Calonectrin. (Dr. Kanaoka's Special issues) 
Akira Iida, Kazuhide Konishi, Hironobu Matsumoto, Masafumi Kaneko, and Kiyoshi Tomioka, Heterocycles, 2003, 59 (2), 595-604.

231. Total Synthesis of $( \pm)$ - $\alpha$ - and $\beta$-Lycoranes by Sequential Chemoselective Conjugate AdditionStereoselective Nitro-Michael Cyclization of $\omega$-Nitro- $\alpha, \beta, \psi, \omega$-unsaturated Ester.

Tomohisa Yasuhara, Katsumi Nishimura, Mitsuaki Yamashita, Naoshi Fukuyama, Ken-ichi Yamada, Osamu Muraoka, and Kiyoshi Tomioka, Org. Lett., 2003, 5 (7), 1123-1126.

232. Initiator Dependent Chemoselective Addition of THF Radical to Aldehyde and Aldimine, and Its Application to Three Component Reaction.

Ken-ichi Yamada, Yasutomo Yamamoto, and Kiyoshi Tomioka, Org. Lett., 2003, 5 (10), 1797-1799.

233. Chiral ligand-controlled catalytic asymmetric epoxidation of $\alpha, \beta$-unsaturated carbonyl compounds with peroxide.

Yoshihito Tanaka, Katsumi Nishimura, and Kiyoshi Tomioka, Tetrahedron, 2003, 59 (25), 4549-4556.

234. Cancer Preventive Potential of Trichothecenes from Trichothecium roseum.

Kazuhide Konishi, Akira Iida, Masafumi Kaneko, Kiyoshi Tomioka, Harukuni Tokuda, Hoyoku Nishino, and Yuko Kumeda, Bioorg. Med. Chem., 2003, 11 (12), 2511-2518.

235. Molecular Assembly of $C_{2}$-Symmetric Bis-(2S)-2-methyldodecanoylamides of $\alpha, \omega-$ Alkylidenediamines into Coiled Coil and Twisted Ribbon Aggregates.

Takaaki Sumiyoshi, Katsumi Nishimura, Minoru Nakano, Tetsuro Handa, Yoshihisa Miwa, and Kiyoshi Tomioka,

J. Am. Chem. Soc., 2003, 125 (40), 12137-12142.

236. Asymmetric alkylation of $N$-toluenesulfonylimines with dialkylzinc reagents catalyzed by copper-chiral amidophosphine.

Takahiro Soeta, Kazushige Nagai, Hidetaka Fujihara, Masami Kuriyama, and Kiyoshi Tomioka, J. Org. Chem., 2003, 68 (25), 9723-9727.

237. Medicinal Chemistry. (translation in Japanese from An Introduction to Medicinal Chemistry, GL. Patrick, Oxford)

Isao Kitagawa, Masakatsu Shibasaki, and Kiyoshi Tomioka (Co ed.), Maruzen, 2003.

238. Asymmetric Conjugate Addition of Arylthiols to Enoates and its Application to Organic Synthesis of Biologically Potent Compounds.

Katsumi Nishimura and Kiyoshi Tomioka, Yakugaku Zasshi, 2003, 123 (1), 9-18.

239. Enantioselective Conjugate Addition and 1,2-Addition to $\mathrm{C}=\mathrm{N}$ of Organolithium Reagents. Mayu Iguchi, Ken-ichi Yamada, and Kiyoshi Tomioka, Topics in Organometallic Chemistry 5. Lithium in Enantioselective Synthesis, Springer, 2003, pp. 37-59.

240. Chiral Ligand-Controlled Asymmetric Reaction of Organolithium. (in Japanese)

Kiyoshi Tomioka, 
New development of Process Chemistry, CMC press, 2003.

241. Unexpected Reaction of a Dimethylzinc-Generated THF Radical with Aldehydes.

Yasutomo Yamamoto, Ken-ichi Yamada, and Kiyoshi Tomioka, Tetrahedron Lett., 2004, 45 (4), 795-797.

242. Chiral Ligand-Controlled Asymmetric Conjugate Addition of $\alpha$-Trimethylsilanylacetate to Acyclic and Cyclic Enones.

Mayu Iguchi, Hirohisa Doi, Seiji Hata, and Kiyoshi Tomioka, Chem. Pharm. Bull., 2004, 52 (1), 125-129.

243. Construction of Arene-Fused-Piperidine Motifs by Asymmetric Addition of 2-Trityloxymethylaryllithiums to Nitroalkenes: the Asymmetric Synthesis of A-86929.

Mitsuaki Yamashita, Ken-ichi Yamada, and Kiyoshi Tomioka, J. Am. Chem. Soc., 2004, 126 (7), 1954-1955.

244. Introduction of Functionalized C1, C2, and C3 Units to Imines through the Dimethylzinc-AirInitiated Radical Addition.

Ken-ichi Yamada, Yasutomo Yamamoto, Masaru Maekawa, and Kiyoshi Tomioka, J. Org. Chem., 2004, 69 (5), 1531-1534.

245. Efficient Synthesis of $( \pm)-\gamma$-Lycorane Employing Stereoselective Conjugate Addition to Nitroolefin.

Tomohisa Yasuhara, Emi Osafune, Katsumi Nishimura, Mitsuaki Yamashita, Ken-ichi Yamada, Osamu Muraoka, and Kiyoshi Tomioka, Tetrahedron Lett., 2004, 45 (15), 3043-3045.

246. Improved Asymmetric Synthesis of Dopamine D1 Full Agonist, Dihydrexidine, Employing Chiral Ligand-Controlled Asymmetric Conjugate Addition of Aryllithium to a Nitroalkene.

Mitsuaki Yamashita, Ken-ichi Yamada, and Kiyoshi Tomioka, Tetrahedron, 2004, 60 (19), 4237-4242.

247. Lithium Amide-Assisted Asymmetric Mannich-Type Reactions of Menthyl Acetate with PMPAldimines.

Seiji Hata, Mayu Iguchi, Tetsuo Iwasawa, Ken-ichi Yamada, and Kiyoshi Tomioka, Org. Lett., 2004, 6 (11), 1721-1723.

248. Catalytic Enhancement Effect of a Chiral Ligand on the Asymmetric Mannich-Type Reactions of Menthyl Alkanoates with Aldimines. (Prof. Teruaki Mukaiyama's Special issue)

Seiji Hata, Tetsuo Iwasawa, Mayu Iguchi, Ken-ichi Yamada, and Kiyoshi Tomioka, Synthesis, 2004, (9), 1471-1475.

249. N-Allyl-N-tert-butyldimethylsilylamine for chiral ligand-controlled asymmetric conjugate addition to tert-butyl alkenoates.

Hirohisa Doi, Takeo Sakai, Ken-ichi Yamada, and Kiyoshi Tomioka, Chem. Commun., 2004, (16), 1850-1851.

250. N-Boc-L-Valine-Connected Amidomonophosphane-Rhodium(I) Catalyst for Asymmetric Arylation of $\mathrm{N}$-Tosylarylimines with Arylboroxines.

Masami Kuriyama, Takahiro Soeta, Xinyu Hao, Qui-An Chen, and Kiyoshi Tomioka, J. Am. Chem. Soc., 2004, 126 (26), 8128-8129.

251. Synthesis of Nitrogen-Functionalized Cyclohexanes using Chemoselective Conjugate Addition of Phenyllithium to Linear $\omega$-Nitro- $\alpha, \beta, \psi, \omega$-Unsaturated Ester and Subsequent Stereoselective 
Intramolecular Nitro-Michael Cyclization.

Tomohisa Yasuhara, Katsumi Nishimura, Emi Osafune, Osamu Muraoka, and Kiyoshi Tomioka, Chem. Pharm. Bull., 2004, 52 (9), 1109-1113.

252. Direct Aminoalkylation of Cycloalkanes through Dimethylzinc-Initiated Radical Process.

Ken-ichi Yamada, Yasutomo Yamamoto, Masaru Maekawa, Jingbo Chen, and Kiyoshi Tomioka, Tetrahedron Lett., 2004, 45 (35), 6595-6597. Cover Figure

253. Structure Tuning of Lithium Amide for Asymmetric 1,4-Addition to Cinnamate and Subsequent Demasking.

Takeo Sakai, Masahiro Doi, Yoshito Kawamoto, Ken-ichi Yamada, and Kiyoshi Tomioka, Tetrahedron Lett., 2004, 45 (50), 9261-9263.

254. Asymmetric Reactions Based on Activation and Structure Control of Molecule -Asymmetric Reaction of Lithiated Nucleophiles-. (in Japanese)

Kiyoshi Tomioka, Yakugaku Zasshi, 2004, 124 (2), 43-53.

255. Industry-university collaboration of process chemistry. (in Japanese)

Kiyoshi Tomioka, Chemical Industry, 2004, 55 (5), 334-337.

256. Radical Reactions Initiated by Diorganozinc.

Ken-ichi Yamada, Yasutomo Yamamoto, and Kiyoshi Tomioka, J. Synth. Org. Chem. Jpn., 2004, 62 (11), 1158-1165.

257. Addition of Organolithium Reagents to Double Bonds.

Hiroshi Yamataka, Ken-ichi Yamada, and Kiyoshi Tomioka, The Chemistry of Organolithium Compounds, Part 1, Chapter 14, ed. by Z. Rappoport, I. Marek, Wiley, 2004.

258. Conjugate Addition of Organometallics to Activated Olefins.

Kiyoshi Tomioka,

Comprehensive Asymmetric Catalyst, Supplement to Chapter 31.1, pp. 109-124, ed. by E. N. Jacobsen, A. Pfaltz, H. Yamamoto, Springer, 2004.

259. Enantioselective Synthesis of Allenes.

Hiroaki Ohno, Yasuo Nagaoka, and Kiyoshi Tomioka, Modern Allene Chemistry, 2nd edn., Part 1, Chapter 4, ed. by Norbert Krause, A. Stephen K. Hashmi, Wiley-VCH, 2004.

260. Organic Chemistry 4th Ed. (Book translation from Paula Yurkanis Bruice, Prentice Hall). Y. Ofune, T. Katsuki, K. Saigo, and K. Tomioka, Co. ed. Kagaku Dojin, 2004.

261. Catalytic Asymmetric Conjugate Addition of Dialkylzinc Reagents to $\beta$-Aryl- $\alpha, \beta$-unsaturated $N$ 2,4,6-Triisopropylphenylsulfonylaldimines with use of $N$-Boc-L-Val-Connected Amidophosphane-Copper(I) Catalyst.

Takahiro Soeta, Masami Kuriyama, and Kiyoshi Tomioka, J. Org. Chem., 2005, 70 (1), 297-300.

262. Dimethylzinc-Initiated Radical Reaction of Cyclic Ethers with Arylamines, Alkoxyamines, and 
Dialkylhydrazines.

Yasutomo Yamamoto, Masaru Maekawa, Tito Akindele, Ken-ichi Yamada, and Kiyoshi Tomioka, Tetrahedron, 2005, 61 (2), 379-384.

263. Thiol-Catalyzed Acyl Radical Cyclization of Alkenals.

Kazuya Yoshikai, Tomoharu Hayama, Katsumi Nishimura, Kenichi Yamada, and Kiyoshi Tomioka, J. Org. Chem., 2005, 70 (2), 681-683.

264. Mechanistic Aspects of Thiyl Radical-Promoted Acyl Radical Cyclization of Formylenoate Cyclization versus Oxidation.

Kazuya Yoshikai, Tomoharu Hayama, Katsumi Nishimura, Ken-Ichi Yamada, and Kiyoshi Tomioka,

Chem. Pharm. Bull., 2005, 53 (5), 586-588. Cover.

265. Chiral Amino Alcohol-Mediated Asymmetric Conjugate Addition of Arylalkynes to Nitroolefins. Mitsuaki Yamashita, Ken-ichi Yamada, and Kiyoshi Tomioka, Org. Lett., 2005, 7 (12), 2369-2371.

266. Chiral Amidophosphane-Copper Catalyzed Asymmetric Conjugate Addition of Dialkylzinc Reagents to Nitroalkenes.

Fanny Valleix, Kazushige Nagai, Takahiro Soeta, Masami Kuriyama, Ken-ichi Yamada, and Kiyoshi Tomioka,

Tetrahedron, 2005, 61 (31), 7420-7424.

267. Asymmetric Synthesis of 5-Arylcyclohexenones by Rhodium(I)-Catalyzed Conjugate Arylation of Racemic 5-(Trimethylsilyl)cyclohexenone with Arylboronic Acid.

Qian Chen, Masami Kuriyama, Takahiro Soeta, Xinyu Hao, Ken-ichi Yamada, and Kiyoshi Tomioka, Org. Lett., 2005, 7 (20), 4439-4441.

268. Catalytic Asymmetric Addition of Terminal Alkynes to Aldehydes Mediated by $(1 R, 2 R)-2-$ (Dimethylamino)-1,2-diphenylethanol.

Mitsuaki Yamashita, Ken-ichi Yamada, and Kiyoshi Tomioka, Adv. Synth. Catal., 2005, 347 (11-13), 1649-1652.

269. Asymmetric Mannich-Type Addition of Lithium Glycolates to Imines Producing 3-Hydroxy-4Phenylazetidin-2-Ones. (Prof. Kenji Koga's Memorial Issue)

Hiroki Fujieda, Seiji Hata, Ken-ichi Yamada, and Kiyoshi Tomioka, Heterocycles, 2005, 66, 611-619.

270. Tin-free Intermolecular Addition of Primary Alkyls to Imines via Dimethylzinc-Air Radical Process.

Ken-ichi Yamada, Yasutomo Yamamoto, Masaru Maekawa, Tito Akindele, Hiroyuki Umeki, and Kiyoshi Tomioka,

Org. Lett., 2006, 8 (1), 87-89.

271. Asymmetric Synthesis of Intermediates for Otamixaban and Premafloxacin by the Chiral Ligand-Controlled Asymmetric Conjugate Addition of a Lithium Amide.

Takeo Sakai, Yoshito Kawamoto, and Kiyoshi Tomioka, J. Org. Chem., 2006, 71 (12), 4706-4709.

272. Chiral Ligand-Controlled Asymmetric Conjugate Amination of Enoates with Lithium 
Mesitylmethyl(trimethylsilyl)amide.

Takeo Sakai, Hirohisa Doi, and Kiyoshi Tomioka, Tetrahedron, 2006, 62 (35), 8351-8359.

273. C2 Symmetric Chiral $N$-Heterocyclic Carbene Catalyst for Asymmetric Intramolecular Stetter Reaction.

Yasumasa Matsumoto and Kiyoshi Tomioka, Tetrahedron Lett., 2006, 47 (33), 5843-5846.

274. Catalytic Asymmetric Reactions Using Environmentally Friendly Reagents And Catalyst. Asymmetric Synthesis vs Kinetic Resolution.

Kiyoshi Tomioka, Pure Appl. Chem., 2006, 78 (11), 2029-2034.

275. Efficient Catalytic Asymmetric Synthesis of trans-5-Aryl-2-substituted cyclohexanones by Rhodium-Catalyzed Conjugate Arylation of Racemic 6-Substituted Cyclohexenones

Qian Chen, Takahiro Soeta, Masami Kuriyama, Ken-ichi Yamada, and Kiyoshi Tomioka, Adv. Synth. Catal., 2006, 348 (18), 2604-2608.

276. Asymmetric Radical Addition of Ethers to Enantiopure $N$ - $p$-Toluenesulfinyl Aldimines, Mediated by Dimethylzinc-Air.

Tito Akindele, Yasutomo Yamamoto, Masaru Maekawa, Hiroyuki Umeki, Ken-ichi Yamada, and Kiyoshi Tomioka,

Org. Lett., 2006, 8 (25), 5729-5732.

277. Enantioselective Conjugate Addition of a Lithium Ester Enolate Catalyzed by Chiral Lithium Amides.

Nicolas Duguet, Anne Harrison-Marchand, Jacques Maddaluno, and Kiyoshi Tomioka, Org. Lett., 2006, 8 (25), 5745-5748.

278. Strategic Applications of Named Reactions in Organic Synthesis. (Book translation from László Kürti, Barbara Czakó, Elsevier Inc.)

Kiyoshi Tomioka, ed.

Kagaku Dojin, 2006.

279. Essential Organic Chemistry (Book translation from Paula Yurkanis Bruice, Prentice Hall).

Y. Ofune, T. Katsuki, K. Saigo, and K. Tomioka, Co. ed. Kagaku Dojin, 2006.

280. Introduction of our laboratory. (in Japanese)

Ken-ichi Yamada, Yasutomo Yamamoto, and Kiyoshi Tomioka, Organometallic News, 2006, 4, 142-143.

281. Peptidic Amidomonophosphane Ligand for Copper-Catalyzed Asymmetric Conjugate Addition of Diorganozincs to Cycloalkenones. (Prof. M. Shibasaki's Special issue)

Takahiro Soeta, Khalid Selim, Masami Kuriyama, and Kiyoshi Tomioka, Adv. Synth. Catal., 2007, 349, 629-635.

282. Kinetic Resolution of 5-Substituted Cycloalkenones by Peptidic Amidophosphane-CopperCatalyzed Asymmetric Conjugate Addition of Diorganozinc.

Takahiro Soeta, Khalid Selim, Masami Kuriyama, and Kiyoshi Tomioka, Tetrahedron, 2007, 63 (28), 6573-6576.

283. Asymmetric Synthesis of 3-Amino-2-hydroxyalkanoates by Mannich Reaction of Menthyl 
Acetate with Imines and Subsequent Oxidation.

Seiji Hata and Kiyoshi Tomioka,

Tetrahedron, 2007, 63 (35), 8514-8520.

284. Catalytic Asymmetric Intramolecular Hydroamination of Aminoalkenes.

Tokutaro Ogata, Atsushi Ujihara, Susumu Tsuchida, Tomoko Shimizu, Atsunori Kaneshige, and Kiyoshi Tomioka,

Tetrahedron Lett., 2007, 48 (38), 6648-6650.

285. Challenging Organic Chemistry. (in Japanese)

Kiyoshi Tomioka,

Farmashia, 2007, 43 (3), 183.

286. Catalysis on Process Development of Pharmaceuticals. (in Japanese)

Toshiaki Mase and Kiyoshi Tomioka, Catalysts \& Catalysis, 2007, 49 (3), 184-188.

287. Chemical Bank. (in Japanese)

Kiyoshi Tomioka and Nobutaka Fujii.

Kagaku, 2007, 62, 5, 18-20.

288. Cumulenes and Allenes, Synthesis by Substitution. (44.2.1)

Hiroaki Ohno and Kiyoshi Tomioka,

Science of Synthesis, 44, 71-174, Houben-Weyl, 2007, Nov. 30.

289. Amidophosphane-Copper(I)-Catalyzed Asymmetric Conjugate Addition of Dialkylzincs to Racemic 6-Substituted Cyclohexenones Giving 2,5-Di- and 2,2,5-Trisubstituted Cyclohexanones. Khalid Selim, Takahiro Soeta, Ken-ichi Yamada, and Kiyoshi Tomioka, Chem. Asian J., 2008, 3, 342-350.

290. Base-induced cyclization-rearrangement of optically active 3-aminoalkanoates to 5- and 7membered lactams.

Takeo Sakai, Ken-ichi Yamada, and Kiyoshi Tomioka,

Chem. Asian J., 2008, 3 (8), 1486-1493.

291. A Ternary Complex Reagent for An Asymmetric Michael Reaction of Lithium Ester Enolates with Enoates.

Yasutomo Yamamoto, Hirokazu Suzuki, Yorinobu Yasuda, Akira Iida, and Kiyoshi Tomioka, Tetrahedron Lett., 2008, 49 (31), 4582-4584.

292. $C_{2}$ Symmetric Chiral NHC Ligand for Asymmetric Quaternary Carbon Constructing CopperCatalyzed Conjugate Addition of Grignard Reagents to 3-Substituted Cyclohexenones.

Yasumasa Matsumoto, Ken-ichi Yamada, and Kiyoshi Tomioka, J. Org. Chem., 2008, 73 (12), 4578-4581.

293. Conjugate addition of THF-2-yl radical with $\alpha, \beta$-unsaturated $N$-tosyl imines using a dimethylzinc-air initiator. Catalytic Asymmetric Intramolecular Allylation of Aldehyde. (Prof. K. Fukumoto's Special issues)

Ken-ichi Yamada, Hiroyuki Umeki, Masaru Maekawa, Yasutomo Yamamoto, Tito Akindele, Mayu Nakano, and Kiyoshi Tomioka,

Tetrahedron, 2008, 64 (30-31), 7258-7265.

294. Copper-Catalyzed Asymmetric Allylic Substitution with Aryl and Ethyl Grignard Reagents.

Khalid Selim, Ken-ichi Yamada, and Kiyoshi Tomioka, 
Chem. Commun., 2008, (41), 5140-5142.

295. Consecutive Cyclization of Allylaminoalkene by Intramolecular Aminolithiation-Carbolithiation. Susumu Tsuchida, Atsunori Kaneshige, Tokutaro Ogata, Hiromi Baba, Yasutomo Yamamoto, and Kiyoshi Tomioka,

Org. Lett., 2008, 10 (16), 3635-3638.

296. Tin-free Radical Addition of Acyloxymethyl to Arylimines.

Ken-ichi Yamada, Mayu Nakano, Masaru Maekawa, Tito Akindele, and Kiyoshi Tomioka, Org. Lett., 2008, 10 (17), 3805-3808.

297. Chemoselective Conjugate Addition of Dimethylzinc-Mediated Ether and Acetal Radicals to Alkylidenemalonates and Asymmetric Reactions. (AIM special)

Ken-ichi Yamada, Masaru Maekawa, Tito Akindele, Mayu Nakano, Yasutomo Yamamoto, and Kiyoshi Tomioka,

J. Org. Chem., 2008, 73 (24), 9535-9538.

298. Copper-catalyzed Asymmetric Alkylation of Imines with Dialkylzinc and Related Reactions.

Ken-ichi Yamada and Kiyoshi Tomioka,

Chemical Review, 2008, 108 (8), 2874-2886.

299. Catalytic Asymmetric Intramolecular Allylation of Aldehyde. (Prof. K. Fukumoto's Special issues)

Tomohiro Sato and Kiyoshi Tomioka, Heterocycles, 2009, 77 (1), 587-593.

300. Aminolithiation of Carbon-Carbon Double Bonds as A Powerful Tool in Organic Synthesis.

Kiyoshi Tomioka, Takeo Sakai, Tokutaro Ogata, and Yasutomo Yamamoto, Pure Appl. Chem., 2009, 81 (2), 247-253.

301. Asymmetric Construction of Quaternary Carbon Centers by Sequential Conjugate Addition of Lithium Amide and in situ Alkylation: Utility in the Synthesis of (-)-Aspidospermidine.

Mayuko Suzuki, Yoshito Kawamoto, Takeo Sakai, Yasutomo Yamamoto, and Kiyoshi Tomioka,

Org. Lett., 2009, 11 (3), 653-655.

302. Total Synthesis of (-)-Lycorine and (-)-2-epi-Lycorine by Asymmetric Conjugate Addition Cascade.

Ken-ichi Yamada, Mitsuaki Yamashita, Takaaki Sumiyoshi, Katsumi Nishimura, and Kiyoshi Tomioka, Org. Lett., 2009, 11 (7), 1631-1633.

303. Tandem Conjugate Addition-Aldol Cyclization of 2-Formylbenzylidenemalonate with Ether Radicals by the Mediation of Dimethylzinc.

Ken-ichi Yamada, Masaru Maekawa, Tito Akindele, Yasutomo Yamamoto, Mayu Nakano, and Kiyoshi Tomioka,

Tetrahedron, 2009, 65 (4), 903-908.

304. Enantioselective Conjugate Addition of a Lithium Ester Enolate Catalyzed by Chiral Lithium Amides: a Possible Intermediate Characterized.

Baptiste Lecachey, Nicolas Duguet, Hassan Oulyadi, Catherine Fressigné, Anne HarrisonMarchand, Yasutomo Yamamoto, Kiyoshi Tomioka, and Jacques Maddaluno,

Org. Lett., 2009, 11 (9), 1907-1910. 
305. Asymmetric Construction of Three Contiguous Stereogenic Centers by Conjugate AdditionAlkylation of Lithium Ester Enolate.

Yasutomo Yamamoto, Yorinobu Yasuda, Hiroyuki Nasu, and Kiyoshi Tomioka, Org. Lett., 2009, 11 (9), 2007-2009.

306. Accessing the Amide Functionality by the Mild and Low-Cost Oxidation of Imine.

Magdi A. Mohamed, Ken-ichi Yamada, and Kiyoshi Tomioka, Tetrahedron Lett., 2009, 50 (26), 3436-3438.

307. Asymmetric Construction of Binaphthyl by the Chiral Diether-Mediated Conjugate Addition of Naphthyllithium to naphthalenecarboxylic acid BHA ester.

Mitsuru Shindo, Yasutomo Yamamoto, Ken-ichi Yamada, and Kiyoshi Tomioka, Chem. Pharm. Bull., 2009, 57 (7), $752-754$.

308. Chiral amidophosphane-rhodium(I)-catalyzed asymmetric conjugate arylation of acyclic enones with arylboronic acids.

Qian Chen, Masami Kuriyama, Xinyu Hao, Takahiro Soeta, Yasutomo Yamamoto, Ken-ichi Yamada, and Kiyoshi Tomioka,

Chem. Pharm. Bull., 2009, 57 (9), 1024-1027.

309. Iron Chloride Enhancement of Dimethylzinc-Mediated Radical Conjugate Addition of Ethers and Amine to Alkylidenemalonates.

Masaru Maekawa, Ken-ichi Yamada, Yasutomo Yamamoto, Mayu Nakano, Tito Akindele, and Kiyoshi Tomioka,

Tetrahedron Lett., 2009, 55 (44), 6040-6043.

310. Steric Tuning of the Amidomonophosphane-Rhodium(I) Catalyst in Asymmetric Addition of Arylboroxines to $\mathrm{N}$-Phosphinoyl Aldimines.

Xinyu Hao, Masami Kuriyama, Qian Chen, Yasutomo Yamamoto, Ken-ichi Yamada, and Kiyoshi Tomioka,

Org. Lett., 2009, 11 (19), 4470-4473.

311. Efficient Chiral NHC-Copper(I)-Catalyzed Asymmetric Allylic Arylation with Aryl Grignard Reagents.

B. Selim, Yasumasa Matsumoto, Ken-ichi Yamada, and Kiyoshi Tomioka, Angew. Chem. Int. Ed., 2009, 48 (46), 8733-8735.

312. Dimethylzinc Initiated Radical Reactions.

Tito Akindele, Ken-ichi Yamada, and Kiyoshi Tomioka, Account of Chemical Research, 2009, 42 (2), 345-355.

313. Organic Chemistry 5th edn. (Book translation from Paula Yurkanis Bruice, Prentice Hall) Y. Ofune, T. Katsuki, K. Saigo, and K. Tomioka, Co. ed. Kagaku Dojin, 2009.

314. Stereoselective Radical Addition of an Acetal unto Sterically Tuned Enantiomerically Pure $N$-Sulfinyl Imines.

Tito Akindele, Ken-ichi Yamada, Takumi Sejima, Masaru Maekawa, Yasutomo Yamamoto, Mayu Nakano, and Kiyoshi Tomioka,

Chem. Pharm. Bull., 2010, 58 (2), 265-269.

315. Chiral Carbene Approach to Gold-Catalyzed Asymmetric Cyclization of 1,6-Enynes. 
Yasumasa Matsumoto, Khalid B. Selim, Hirotsugu Nakanishi, Ken-ichi Yamada, Yasutomo Yamamoto, and Kiyoshi Tomioka,

Tetrahedron Lett., 2010, 51 (2), 404-406.

316. NMR Studies of a Ternary Complex Reagent of Lithium Ester Enolate, Chiral Diether, and Lithium Diisopropylamide in an Asymmetric Michael Reaction.

Yasutomo Yamamoto, Yorinobu Yasuda, Hassan Oulyadi, Jacques Maddaluno, and Kiyoshi

Tomioka, Tetrahedron, 2010, 66 (13), 2470-2473.

317. Stereoselective Formal Synthesis of (+)-Allokainic Acid via Thiol-mediated Acyl Radical Cyclization.

Ken-ichi Yamada, Tomohiro Sato, Masaki Hosoi, Yasutomo Yamamoto, and Kiyoshi Tomioka, Chem. Pharm. Bull., 2010, 58 (11), 1511-1516.

318. Chiral N-Heterocyclic Carbene-Copper(I)-Catalyzed Asymmetric Allylic Arylation of Aliphatic Allylic Bromides: Steric and Electronic Effects on $\gamma$-Selectivity.

Khalid B. Selim, Hirotsugu Nakanishi, Yasumasa Matsumoto, Yasutomo Yamamoto, Kenichi Yamada, and Kiyoshi Tomioka,

J. Org. Chem., 2011, 76 (5), 1398-1408.

319. Rhodium-Catalyzed Asymmetric Phenylation of N-Phosphinoylarylimines with Triphenylborane.

Hao Xinyu, Qian Chen, Masami Kuriyama, Ken-ichi Yamada, Yasutomo Yamamoto, and Kiyoshi Tomioka,

Catal. Sci. Tech., 2011, 1 (1), 62-64.

320. Steric influence of $N$-phosphorus-arylimines on the rhodium-catalyzed asymmetric arylation (Prof. Satoshi Omura special).

Hao Xinyu, Qian Chen, Ken-ichi Yamada, Yasutomo Yamamoto, and Kiyoshi Tomioka, Tetrahedron, 2011, 67 (35), 6469-6473.

321. Crosscoupling. (in Japanese)

Yasutomo Yamamoto and Kiyoshi Tomioka, Clinician, 2011.

322. Let's Apply Process Chemistry for Drug Discovery. (in Japanese)

Kiyoshi Tomioka, J. Synth. Org. Chem. Jpn, 2011, 69 (5), 483.

323. Steric tuning of $\mathrm{C}_{2}$-symmetric chiral $\mathrm{N}$-heterocyclic carbene in gold-catalyzed asymmetric cyclization of 1,6-enynes

Ken-ichi Yamada, Yasumasa Matsumoto, Khalid B. Selim, Yasutomo Yamamoto, and Kiyoshi Tomioka,

Tetrahedron, 2012, 68 (22), 4159-4165.

324. High Performance of $N$-Alkoxycarbonyl-Imines in Triethylborane-Mediated Tin-Free Radical Addition.

Ken-ichi Yamada, Takehito Konishi, Mayu Nakano, Shintaro Fujii, Romain Cadou, Yasutomo Yamamoto, and Kiyoshi Tomioka, J. Org. Chem., 2012, 77 (3), 1547-1553. 
325. General Entry to Asymmetric One-Pot $[N+2+n]$ Cyclization for the Synthesis of Three- to Seven-Membered Azacycloalkanes. Featured Article

Shingo Harada, Takeo Sakai, Kiyosei Takasu, Ken-ichi Yamada, Yasutomo Yamamoto, and Kiyoshi Tomioka,

J. Org. Chem., 2012, 77 (17), 7212-7222.

326. Total Synthesis of (-)-Kopsinine by an Asymmetric One-Pot [N+2+3] Cyclization.

Shingo Harada, Takeo Sakai, Kiyosei Takasu, Ken-ichi Yamada, Yasutomo Yamamoto, and Kiyoshi Tomioka,

Chem. Asian J., 2012, 7 (10), 2196-2198.

327. Catalytic Asymmetric Synthesis of $(S)$-Laudanosine by Hydroamination. (Prof. Negishi's Special issue)

Tokutaro Ogata, Tetsutaro Kimachi, Ken-ichi Yamada, Yasutomo Yamamoto, and Kiyoshi Tomioka, Heterocycles, 2012, 86 (1), 469-485

328. Radical One-Pot $\alpha, \beta$-Dual and $\beta$-Mono Oxymethylation of Alkylidenemalonate.

Ken-ichi Yamada, Takehito Konishi, Mayu Nakano, Shintaro Fujii, Romain Cadou, Yasutomo Yamamoto, and Kiyoshi Tomioka, J. Org. Chem., 2012, 77 (13), 5775-5780.

329. Total Synthesis of (+)-trans-Dihydronarciclasine Utilizing Asymmetric Conjugate Addition.

Ken-ichi Yamada, Yuzo Mogi, Magdi A. Mohamed, Kiyosei Takasu, and Kiyoshi Tomioka, Org. Lett., 2012, 14 (23), 5868-5871.

330. Unexpected Process Chemistry of Pharmaceuticals-Asymmetric Reaction of Racemic Starting Materials. (in Japanese)

Kiyoshi Tomioka, Plant and Process, 2012, 7, 17-20.

331. Stoichiometric Auxiliary Ligands for Metals and Main Group Elements: Ligands for Lithium.

Kiyoshi Tomioka, Yasutomo Yamamoto, and Ken-ichi Yamada, in Comprehensive Chirality, Volume 3, pp. 626-654, ed. by E. M. Carreira and H. Yamamoto, Amsterdam: Elsevier, 2012, Oct. 15.

332. C-C Bond Formation (Transition Metal-Catalyzed Michael).

Kiyoshi Tomioka, Yasutomo Yamamoto, and Ken-ichi Yamada, in Comprehensive Chirality, Volume 4, pp. 122-131, ed. by E. M. Carreira and H. Yamamoto, Amsterdam: Elsevier, 2012 Oct 15.

333. Critical Profiles of Chiral Diether-Mediated Asymmetric Conjugate Aminolithiation of Enoate with Lithium Amide as a Key to the Total Synthesis of (-)-Kopsinine.

Shingo Harada, Takeo Sakai, Kiyosei Takasu, Ken-ichi Yamada, Yasutomo Yamamoto, and Kiyoshi Tomioka, Tetrahedron, 2013, 69 (15), 3264-3273.

334. NMR Studies on the Structure of a Lithium Amide-Chiral Diether Complex for an Asymmetric Reaction.

Yasutomo Yamamoto, Hiroyuki Nasu, and Kiyoshi Tomioka, Tetrahedron, 2013, 69 (18), 3836-3840.

335. One-Pot Synthesis of $N$-Heterocycles by a Tandem Addition Reaction of $\omega$-Alkenylmagnesium 
Bromide with $\mathrm{C}=\mathrm{N}$ Double Bond-Oxidative Bromolactamization.

Yasutomo Yamamoto, Yuji Takahama, Misa Shimizu, Ai Ohara, Akari Miyawaki, and Kiyoshi Tomioka, Tetrahedron Lett., 2013, 54 (32), 4313-4315.

336. One-Pot Construction of Nitrogen-containing Heterocycles using Anionic Species. (in Japanese)

Kiyoshi Tomioka, Plant and Process, 2013, 7, 45-48.

337. Addition and in situ Halo-Cyclization of $\omega$-Alkenylmagnesium Halide with Aldehydes, Ketone, Carbon Dioxide, and Azodicarboxylate.

Yasutomo Yamamoto, Misa Shimizu, Ai Ohara, Akari Miyawaki, and Kiyoshi Tomioka, New J. Chem., 2013, 37 (12), 3873-3876.

338. Total Synthesis of Javaberine A and 8-epi-Javaberine A. (Prof. Snieckus's Special issue)

Yasutomo Yamamoto, Yuri Tabuchi, Ayana Baba, Kumiko Hideshima, Mai Nakano, Akari Miyawaki, and Kiyoshi Tomioka, Heterocycles, 2014, 88 (2), 1311-1321.

339. Copper-Catalyzed Asymmetric Addition Reaction of Imines.

Kiyoshi Tomioka, Ken-ichi Yamada, and Yasutomo Yamamoto

in Copper-Catalyzed Asymmetric Synthesis, pp. 239-266, ed. by A. Alexakis, N. Krause, and S. Woodward, Wiley, 2014, Jan.

340. Contiguous Radical Pivaloyloxymethylation-directed $\mathrm{C}\left(\mathrm{sp}^{3}\right)-\mathrm{H}$ Iodination of $N$-Tosyl cycloalkanecarboaldimine. (Wasserman's Special issue)

Shintaro Fujii, Mayu Nakano, Yousuke Yamaoka, Kiyosei Takasu, Ken-ichi Yamada, and Kiyoshi Tomioka, Tetrahedron Lett., 2015, 56 (23), 3086-3089.

341. A Short-step Synthesis of (+)- $\beta$-Lycorane by Asymmetric Conjugate Addition Cascad. (Prof. Katrizky's Special)

Katsumi Nishimura, Naoshi Fukuyama, Tomohisa Yasuhara, Mitsuaki Yamashita, Takaaki Sumiyoshi, Yasutomo Yamamoto, Ken-ichi Yamada, and Kiyoshi Tomioka, Tetrahedron, 2015, 71 (39), 7222-7226.

342. Cyclic Model for the Asymmetric Conjugate Addition of Organolithium with Enoate.

Katsumi Nishimura, Naoshi Fukuyama, Mitsuaki Yamashita, Takaaki Sumiyoshi, Yasutomo Yamamoto, Ken-ichi Yamada, and Kiyoshi Tomioka, Synthesis, 2015, 47 (15), 2256-2264.

343. Exploration of Dimethylzinc-Mediated Radical Reactions.

Ken-ichi Yamada and Kiyoshi Tomioka, Chemical Record, 2015, 15 (5), 854-871.

344. Enhancement of Self-Assembly and Gelation Ability of $N, N$ '-Didodecanoyl Ethylenediamine Organogelator by Terminal Functionalization.

Yasutomo Yamamoto, Akane Oyanagi, Akari Miyawaki, and Kiyoshi Tomioka, Tetrahedron Lett., 2016, 57 (52), 5889-5892.

345. Design, Synthesis, and Evaluation of DNA Topoisomerase II-Targeted Nucleosides.

Hironobu Matsumoto, Mitsuaki Yamashita, Teruyuki Tahara, Shinya Hayakawa, Shun-ichi Wada, Kiyoshi Tomioka, and Akira Iida,

Bioorg. Med. Chem., 2017, 25 (15), 4133-4144. 
346. Consecutive Aminolithiation-Carbolithiation of Linear Aminoalkene Bearing Terminal Vinyl Sulfide Moiety Giving Hydroindolizine.

Yasutomo Yamamoto, Tatsuya Yamaguchi, Atsunori Kaneshige, Aiko Hashimoto, Sachiho Kaibe, Akari Miyawaki, Ken-ichi Yamada, and Kiyoshi Tomioka, Synlett, 28 (20), 2913-2917.

347. New Horizons of Process Chemistry: Scalable Reactions and Technologies.

Kiyoshi Tomioka, Takayuki Shioiri, and Hironao Sajiki (Co. ed.)

Springer, (2017, 03 27).

348. Aminolithiation-arylation consecutive cyclization of $N$-(2-fluorophenyl)methylaminoalkylstyryls giving aryl-substituted pyrido[1,2- $b$ ]isoquinolines.

Yasutomo Yamamoto, Yasue Nakanishi, Ken-ichi Yamada, and Kiyoshi Tomioka, Tetrahedron, 2018, in press. 